\title{
A Decade of Mighty Lipophagy: What We Know and What Facts We Need to Know?
}

\author{
Muhammad Babar Khawar $\mathbb{D}^{1},{ }^{1}$ Muddasir Hassan Abbasi ${ }^{\circ},{ }^{2}$ Mussarat Rafiq $\left(\mathbb{D},{ }^{3}\right.$ \\ Naila Naz ${ }^{\left(\mathbb{D},{ }^{4}\right.}$ Rabia Mehmood ${ }^{(D)}{ }^{3}$ and Nadeem Sheikh ${ }^{3}{ }^{3}$ \\ ${ }^{1}$ Molecular Medicine and Cancer Therapeutics Lab, Department of Zoology, Faculty of Sciences, University of Central Punjab, \\ Lahore, Pakistan \\ ${ }^{2}$ Department of Zoology, University of Okara, Okara, Pakistan \\ ${ }^{3}$ Cell \& Molecular Biology Lab, Department of Zoology, University of the Punjab, Q-A-Campus, Lahore 54590, Pakistan \\ ${ }^{4}$ Division of cell Matrix Biology and Regenerative Medicine, University of Manchester, Manchester, UK
}

Correspondence should be addressed to Nadeem Sheikh; nadeem.zool@pu.edu.pk

Received 22 February 2021; Revised 30 September 2021; Accepted 15 October 2021; Published 5 November 2021

Academic Editor: Carla Tatone

Copyright (C) 2021 Muhammad Babar Khawar et al. This is an open access article distributed under the Creative Commons Attribution License, which permits unrestricted use, distribution, and reproduction in any medium, provided the original work is properly cited.

\begin{abstract}
Lipids are integral cellular components that act as substrates for energy provision, signaling molecules, and essential constituents of biological membranes along with a variety of other biological functions. Despite their significance, lipid accumulation may result in lipotoxicity, impair autophagy, and lysosomal function that may lead to certain diseases and metabolic syndromes like obesity and even cell death. Therefore, these lipids are continuously recycled and redistributed by the process of selective autophagy specifically termed as lipophagy. This selective form of autophagy employs lysosomes for the maintenance of cellular lipid homeostasis. In this review, we have reviewed the current literature about how lipid droplets (LDs) are recruited towards lysosomes, cross-talk between a variety of autophagy receptors present on LD surface and lysosomes, and lipid hydrolysis by lysosomal enzymes. In addition to it, we have tried to answer most of the possible questions related to lipophagy regulation at different levels. Moreover, in the last part of this review, we have discussed some of the pathological states due to the accumulation of these LDs and their possible treatments under the light of currently available findings.
\end{abstract}

\section{Autophagy: A Brief Overview}

Autophagy is a conserved intracellular degradative process conserved from very simple organisms (yeast) to highly complex animals (mammals). This process begins with the formation of a double-membrane cup-like structure named phagophore in response to stress circumstances (i.e., nutrient scarcity). In the subsequent step, autophagosome is formed as a result of an extension of the phagophore, sequestrate of cytoplasmic contents/organelles, and finally, the closure of both ends. Afterward, the resultant autophagosome is fused with the lysosome to form an autolysosome to finally break down the desired substance (Figure 1) [1-3]. Autophagy is not only important for supplying energy to starving cells; instead, it is very crucial for the smooth physiological integrity of the cells. Autophagy-defects or deficiency may result in several ailments including neurodegenerative disorders, cardiac diseases, diabetes $[4,5]$, reproductive abnormalities, and even infertility [6].

The research on autophagy progressed with an outstanding pace, and to date, an ample number of autophagy-related (Atg) genes (more than 30) have been identified. There are four key events involved in autophagosome formation: (1) initiation: translocation of uncoordinated 51-like kinase 1 (ULK1) complex upon the mammalian target of rapamycin (mTOR) results in class III phosphatidylinositol 3-kinase (PI3K) activation that ultimately helps in phosphatidylinositol 3-phosphate (PI3P) formation and PI3P-binding proteins recruitment at 


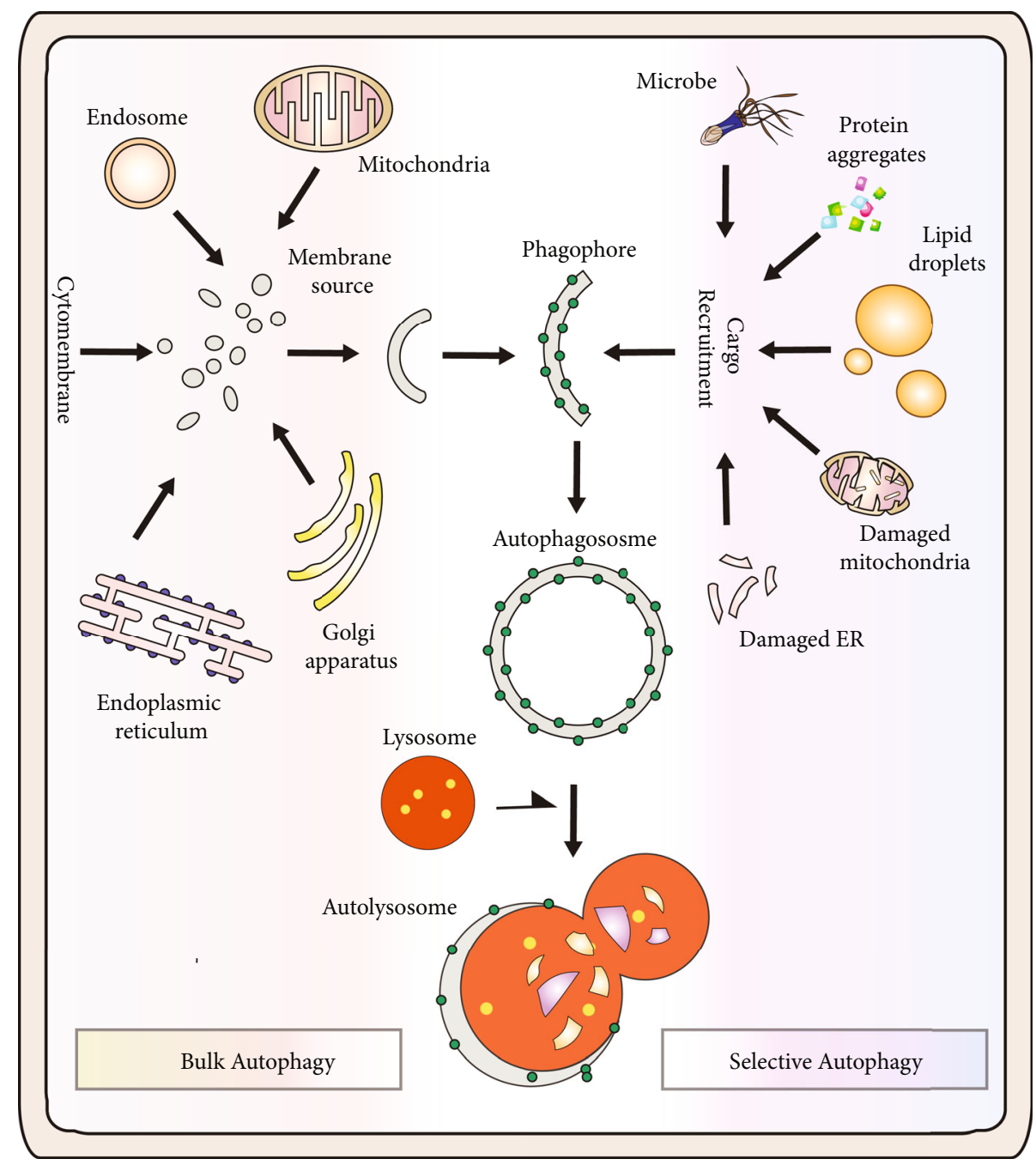

FIgURE 1: Bulk autophagy vs. selective autophagy: autophagy is a natural intracellular catabolic activity that helps cell in the removal of unwanted substances and recycling of cellular components while selective autophagy selectively target several specific cargos including various organelles, cellular substances, and protein aggregates.

the site of autophagosome formation. (2) Vesicle nucleation: Atg9L1 (a transmembrane protein) is involved in the nucleation event of the autophagosomal membrane. (3) Autophagosome formation: this event involved the conjugation of C-terminus of microtubule-associated protein 1A/1Blight chain 3 (LC3) with phosphatidylethanolamine (PE) for anchoring of the protein in the membrane and is mediated by a ubiquitin-like system (Atg7, Atg3, and the Atg12-Atg5-Atg16L1 complex). (4) In the final step, the isolated membrane is elongated and degraded to help the fusion of this autophagosome with lysosome $[3,7,8]$.

\section{Selective Autophagy}

Autophagy triggered by food restriction was formerly considered to be a nonselective process, but a growing body of data suggests that it is, since autophagy selectively targets a variety of cargos, including organelles and protein aggregates (Figure 1). "Autophagy cargo receptors" are the main players of selective autophagy as they bind with the targeted mate- rials having degradation signals (ubiquitin) via ubiquitinbinding domain (UBD). In mammals, the presence of an LC3 interacting region (LIR) motif equips these receptors with a unique property to interact with LC3 (Atg8) present on newly formed autophagosomes. As a result, by serving as molecular bridges, these autophagy cargo receptors designated the targeted compounds, tagged with a ubiquitin tag, for destruction by autophagy or the ubiquitin proteasome system (UPS). These receptors, such as p62/ SQSTM1, NBR1, and histone deacetylase 6 (HDAC6), support the removal and destruction of protein aggregates via UBD and LIR2 in aggrephagy (a form of selective autophagy) [3, 7-10]. Despite the lack of orthologues of UBD-containing adaptor proteins (p62) in yeast, mass spectrometric studies recently assisted in the identification of Cue5, which is likely a possible autophagy cargo receptor [11]. There is a coupling of ubiquitin conjugation to ER degradation (CUE) domain in yeast Cue5 (similar to UBDs in mammals) that can interact with Atg8 and binds to ubiquitylated targets. Therefore, Cue 5 helps in the clearance and removal of ubiquitylated 
cargo via selective autophagy by acting as an ubiquitin-Atg8 adaptor protein (analogous to p62 in mammals). Yeast Cue5 and TOLLIP (mammalian orthologue) both aim to remove aggregation-prone proteins (i.e., huntingtin), unable to degrade via UPS, by autophagy (K. [11]). Besides the removal of protein aggregates, selective autophagy also helps in the clearance of damaged organelles (Figure 1). For instance, selective autophagic degradation of worn out and superfluous mitochondria is specifically named as mitophagy [12, 13]. Similarly, the degradation and removal of peroxisomes are known as pexophagy $[14,15]$. In mammals, LC3positive phagophores are recruited, by p62 and NBR1 (autophagy cargo receptors), to the surface of targeted monoubiquitylated peroxisomes [16, 17]. Besides mitophagy and pexophagy, selective autophagy can be categorized into various types depending upon the specific cargos to be targeted, i.e., aggrephagy, xenophagy, glycophagy, reticulophagy, ribophagy, nucleophagy, zymophagy, and lipophagy. Unlike mitochondria and peroxisomes, the detailed insight of molecular mechanism and the role of selective autophagy in the clearance of other organelles need to be elucidated. Intriguingly, ER degradation in yeast seems to be accomplished via microautophagy but in fact, no microautophagy machinery is involved in this process [18]. Moreover, largesized macromolecules like iron complexes and lipids can also be degraded by selective autophagy. Similarly, several intermediary macromolecular species, i.e., inflammasome, midbody, and midbody ring, are also targeted by selective autophagy [19-22]. However, the only lipophagy will be discussed in the rest of this review.

\section{Lipophagy: A General Overview}

There is a battery of powerful hydrolytic enzymes in lysosomes including glycases, nucleases, proteases, and lipases that are responsible for a rapid turnover of unnecessary cell components [23]. Molecular basis of how cellular fat stores are regulated in lipid droplets (LDs) remains a hot topic in the past few years [24]. Cells tend to store the surplus energy in the form of fats, during nutrient-rich status, within special cytoplasmic structures called LDs that act as a vast depot of a variety of molecules such as esters, cholesteryl esters, neutral lipids, and triglycerides [25]. These LDs are encircled by a variety of coat proteins (generally perilipins family proteins (PLINs)) and a single layer of phospholipids [26]. These fat depots are often regarded as distinct organelles because of their ability to neutralize the effects of infectious entities [27] and misfolded proteins [28] and also because of their dynamic interactions with other cytoplasmic organelles [29]. LDs are manufactured at ER and generally range in size from 0.1 to $10 \mu \mathrm{m}$ in most of the cell types [25, 30]. However, LDs found in adipocytes (fat storage cells) may attain a much larger size than average and grow up to $100 \mu \mathrm{m}$ in size [31].

Starvation stimulates lipids to break down in a process named as lipolysis (Figure 2) by protein kinase A activation that results in phosphorylation of PLIN1 (LD coat protein) [32]; this phosphorylation helps in degradation of PLIN1 by proteasomal activity that recruits cytosolic lipases to
LDs [32]. The autophagolysosomal system was postulated to be implicated in the destruction of LDs during starvation because nutritional shortage causes autophagy activation, which supplies nutrients by breaking down worn out and unneeded cytoplasmic material via lysosomal degradation. Hepatocytes were either chemically treated with an autophagy inhibitor (3-methyladenine) or autophagy was inhibited using small hairpin (sh) RNA against Atg5 to test this hypothesis. Following that, in both situations of autophagy failure, a substantial rise in LD content was found [22]. Moreover, an increased level of triglyceride was also observed in autophagy-deficient mouse embryonic fibroblasts [22]. Further studies employing mitochondrial $\beta$-oxidation and rates of triglyceride breakdown revealed this increase in lipid contents was a result of decreased lipid turnover in autophagy-deficient cells instead of an increased triglyceride synthesis. In addition to it, sequestration of LDs by LC3 (autophagy marker)-labeled autophagosomal membrane was confirmed using immunogold electron microscopy. Subsequently, in the starved liver, the presence of LC3, PLIN1, and PLIN2 was also confirmed in LDs and lysosomes [22]. Furthermore, the colocalization of LDs and lysosomes indicated the significance of the lysosomal degradation system in LD catabolism [22]. As autophagosomes were found to engulf LDs and subsequently fused with lysosomes, lipophagy is regarded as selective macroautophagy (macrolipophagy) involved in LD breakdown (Figure 2). In line with these findings, selective Atg7-knock out in the liver resulted in increased LD contents in vivo and was comparable to nonalcoholic fatty liver disease (humans) histologically [22]. Interestingly, the acute supply of lipids (oleic acid) was found to trigger lipophagy in hepatocytes [22]. Lipophagy (autophagy) activation is perhaps an ubiquitous response against a sharp lipid influx as observed in various cell types. For instance, similar response to eliminate the excessive lipid contents was also observed in cultured hypothalamic neurons treated with oleic acid [33]. In contrast to the acute supply of lipids, chronic lipid supply showed an opposite trend, and suppression of lipophagy was observed in mice obesity models (both genetic and diet-fed models) and led to ER stress and hepatic inflammation upon prolonged high-fat-diet feeding [22]. In line with it, ATG7overexpression successfully reversed hepatic LD accumulation and prevented steatosis in obese mice [34]. The results of all these studies are suggestive of an important role of lipophagy (autophagy) in LD turnover. However, an outstanding question that what causes the autophagic flux to decrease under over nutrition conditions remains to be elucidated. Multiple mechanisms are speculated to be involved in decreasing the autophagy in obesity. Previously, in vivo assay showed a $70 \%$ decrease in the fusion of autophagosome with lysosome in a high-fat diet mice model [35]. Similarly, a significant decrease in autophagolysosomal fusion was also noted in alcohol-induced hepatotoxicity [36]. Besides lysosomal pH and ATP availability, few dietary factors also affect autophagolysosomal fusion activity by altering the lipid composition of the lysosomal membrane [35, 37]. The first report describing autophagy contribution in hepatic LD turnover threw into gear a new chapter of 


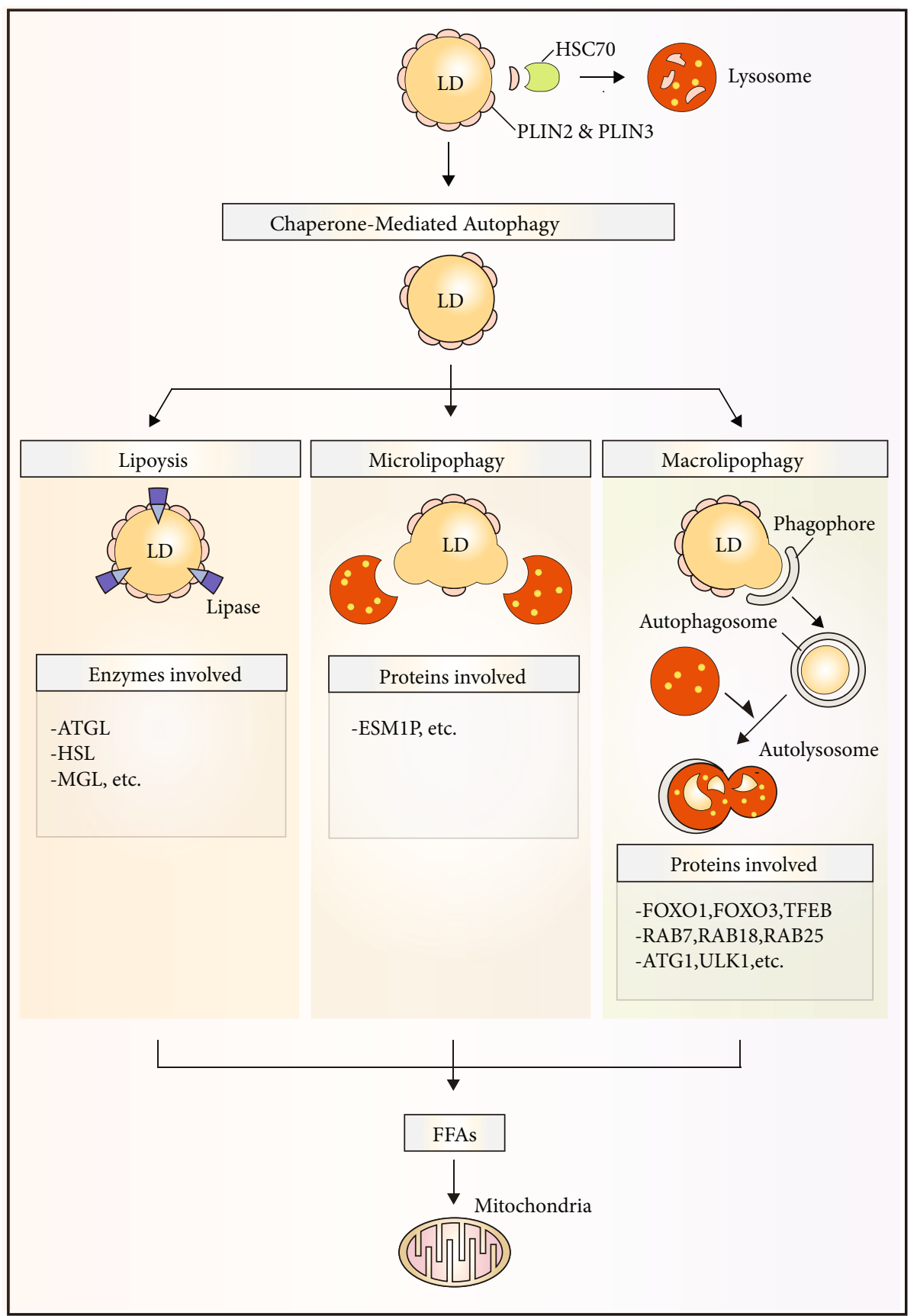

FIGURE 2: Major pathways in LD degradation: CMA facilitates LD degradation by promoting PLIN protein degradation allowing access for lipases. Moreover, both microlipophagy and macrolipophagy also degrade LDs to generate free FAs that are utilized in mitochondria for their complete oxidation.

research, and since that time, a plethora of studies have now identified lipophagy as the main player involved in LD breakdown in a variety of cells, i.e., adipose-resident macrophages [38], prostate cancer cells [39], orexigenic hypothalamic neurons [33], lymphocytes [40], cultured adipocytes [41], macrophage foam cells [41-43], primary striatal neurons, glial cells [44], and gastrointestinal epithelial cells [45]; in nonmammals, i.e., yeast [46, 47], fungus [48], and worms [49, 50]; in plants including rice [51]; and also in phyllosphere microorganisms [52]. Therefore, these findings are enough to support the use of term macrolipophagy/lipophagy as an ubiquitous pathway responsible for LD mobilization [22].

Lipophagy is a highly regulated process; recently, autophagy was found to be regulated by SNARE (soluble N-ethylmale-imide-sensitive factor-attachment protein receptors) proteins that do so by facilitating the recruitment of ATG proteins to autophagosomal formation sites $[53,54]$. SNARE protein syntaxin-17 has also been reported to regulate autophagosomal fusion with lysosomes; however, whether 
this protein is also involved in decrease of lipophagy/autophagolysosomal fusion in obesity is still not known [53]. Moreover, membrane GTPases have been associated with LD degradation as LDs were found to be significantly accumulated in autolysosomes upon small GTPase dynamin-2 inhibition [55]. Recently, Bif-1 (a membrane curvature protein) has been identified as a novel protein regulating lipophagy in adipocytes (Y. [56]). Lipophagy induced an enhanced Bif-1-dependent PLIN1 breakdown, and its deficiency ultimately reduced triacylglycerol (TAG) hydrolysis, suggesting the significance of Bif-1 in lipophagy-dependent PLIN1 degradation and subsequently LD catabolism (Y. [56]). Interestingly, membrane curvature was shown to vary with the size of the LDs; however, whether Bif- 1 regulates lipophagy via altering the size of the LDs is unknown at this time. In fact, the processes that activate lipophagy may be context-dependent and differ depending on the cell type. These lipophagic processes are adapted by cells to cope with severe conditions such as starvation and lipotoxicity. Despite these differences in processes, it is assumed that the basic machinery for lipophagic breakdown is conserved [57].

\section{Mechanisms of Lipophagy Induction and Its Molecular Machinery}

4.1. PLINs and Lipases in Lipophagy Induction. Despite variations in the size, composition, and location of LDs in the cell, the best feature that better defines their dynamic character is their proteome. On the surface of the LDs are a number of proteins that control their signalling and metabolization. One of these proteins' characteristics is their participation in the control of LD-related processes, particularly lipophagy. The perilipin family of proteins is one of the bestknown protein families that affect LD activities and perform many tasks in LD biology [58]. PLIN family comprised of five members (PLIN 1-5) that share the varying degree of homology and differs from each other in their activities and tissue expression profiles. PLINs are capable of modulating the access of lipases to LD surface, hence regulating the LD metabolism [58]. It has been recently reported that PLINs are the major molecules that may link chaperonemediated autophagy (CMA) to LD catabolism [59]. All of these investigations have described the accumulation of LDs upon lysosome-associated membrane protein 2A (LAMP2A) degradation which is an important protein involved in CMA. Moreover, to promote CMA-mediated breakdown in lysosomes, the binding of the heat shock cognate protein of $70 \mathrm{kDa}(\mathrm{Hsc} 70)$ to $\mathrm{CMA}$ recognition motif (KFERQ) within PLIN2 and PLIN3 was observed (Figure 2). Reduction of lipophagy and lipolysis mediated by lipases was found to be decreased upon CMA ablation and is suggestive of the significance of PLIN2 and PLIN3 degradation in promoting LD catabolism and in allowing access of adipose triglyceride lipase (ATGL) and other autophagic proteins to LD surface. Further investigation helped in the identification of a vital protein named AMPactivated protein kinase that is involved in CMA-mediated degradation of phosphorylated PLIN2 [60]. As a result of these findings, it can be inferred that PLIN degradation through CMA is an upstream process necessary for lipophagy start. The importance of ATGL in the catabolism of TAG during the degradation of PLINs may also be deduced from the aforementioned explanation. More in-depth research later revealed a strong connection between autophagy (lipophagy) and ATGL. For instance, a direct interaction between LC3 and ATGL has been demonstrated on the LD surface [61] along with LC3 interactions with HSL. This interaction between LC3 and ATGL occurs at LIR residues 145-150 (STFIPV). LIR depletion caused a deficiency in ATGL localization and translocation to the LD surface during hunger. These findings point to LC3 playing a crucial role in TAG hydrolysis by transporting ATGL to the LD surface. However, it is uncertain why ATGL requires LC3 for its localization to the LD surface.

According to the studies carried out to date, it can be perceived that both ATGL and lipophagy contribute directly to LD catabolism (Figure 2). Sathyanarayan and colleagues have recently described if there any linearity exists between ATGL and autophagy/lipophagy [62]. ATGL was found to promote the interactions of LC3 and lysosomes with LDs and positively regulates autophagic flux in the liver suggesting enhanced lipophagy. Intriguingly, the effects of ATGL overexpression on LD breakdown were diminished as a result of lysosomal acid lipase (LAL) degradation or genetic/chemical inhibition of autophagy. ATGL was previously shown to promote SIRT1 (an important autophagy player) activity $[63,64]$, and SIRT1 was also found to mediate the effects of ATGL in endorsing autophagy (lipophagy). Furthermore, we recently reported on Sirtl's function in testosterone production via autophagy control in the LC3dependent pathway. Sirt1 promotes autophagosome production by deacetylating LC3 in the nucleus of leydig cells. NHERF2 is degraded by autophagosomes, which increases the expression of SR-BI. By increasing cholesterol absorption, SR-BI speeds up testosterone biosynthesis [65].

Subsequently, autophagy (lipophagy) was found to be increased by PNPLA5 and PNPLA8 (two members of ATGL (PNPLA2) containing family) in the variety of cell types [66, 67]. It has been described that diacylglycerol produced by PNPLA5 is involved in the synthesis of autophagosomal membranes, hence regulating protein trafficking by influencing membrane curvatures. Concluding the above results, it can be interpreted that lipases including ATGL regulate autophagy (lipophagy) and the resultant lipophagy mediates bulk degradation of LDs. It is noteworthy that impaired lipophagy and normal macroautophagy observed in macrophages lacking ATGL are indicative of the existence of celltype-specific function [68]. Although lipolysis is tightly regulated via multiple signaling pathways (cAMP/PKA, AMPK, etc.) and a vast variety of proteins influence it via direct interaction with ATGL or other LD proteins, much remains to investigate about how lipophagy is regulated via ATGLmediated signaling.

4.2. Rab GTPases in Lipophagy Induction. Many Rab proteins have been known to play critical roles in LD metabolism and LD biology since the time of their discovery [69-72]. Around 70 members of Rab family GTPases are 
thought to regulate endosomal membrane trafficking via conferring a special "identity" to trafficking items [73]. Rabs serve as molecular switches, cycling between active (GTPbound) and inactive (GDP-bound) states. By controlling the connections between membrane fusion complexes and cytoskeletal components, this interconversion is critical for enabling the complicated intracellular vesicular traffic (motor proteins). The discovery of over 30 Rab GTPases on the surface of LDs has sparked a fresh wave of research into their role in LD catabolism [74]. An impaired lipophagy, characterized by a pronounced decrease in LD turnover, was observed upon the ablation of several members of the GTPases family. Rab7 is an important player in autophagosomal maturation $[75,76]$ and intracellular trafficking (a marker of the endocytic pathway) [77], one of those Rabs that concentrate on LD surface. Rab7 facilitates the interactions between components of HOPS tethering complex and numerous SNARE proteins hence promote the fusion of endocytic and autophagic membranes [78, 79]. Moreover, Rab7, along with several downstream effector components including FYVE, FYCO1, and RILP, mediates organelle transport (both plus- and minus-end directed) in tight cooperation with kinesin and dynein-dynactin motors [80-82]. Therefore, Rab7 is thought to play a prime role in mediating interactions among many cell components and actively contributes to promoting versatile biological activities such as autophagy (lipophagy). Interestingly, Rab7 has also been found to play an important role in mitophagy where it facilitates the encapsulation of mitochondria within autophagosomes with the help of TBC1D15 and TBC1D17 (two significant GTPase-activating proteins) [83]. Rab7's enhanced colocalization with LDs, lysosomes, and autophagosomal membranes led to the discovery of Rab7's important involvement in LD homeostasis for the first time [84]. Rab7-knockdown via siRNA or a negative form of Rab7 in hepatoma cells led to an abnormal LD accretion [85]. During starvation, Rab7 facilitates the priming of LDs by increasing GTP affinity than GDP and recruiting the degradative machinery to $\mathrm{LD}$ surface by directly activating on LD surface [86]. The findings of this study point to Rab7's importance in regulating hepatic lipophagy by serving as an LD-localized node. Rab7 has recently been linked to ethanol-induced hepatic steatosis as well [87]. Rats on an alcohol-rich diet were found to be resistant to starvationinduced lipophagy and developed hepatic steatosis as a result. Rab7 levels were substantially lower in hepatocytes treated with alcohol compared to controls, indicating an autophagic failure in LD detection. Furthermore, more research is needed to determine the specific mechanism behind the inhibition of lipophagy in ethanol-exposed hepatocytes. Rab10, which is found on the surface of LDs, is another member of the Rab family that may play a role in lipophagy. This Rab member regulates Golgi trafficking during epithelial polarization [88] and insulin-stimulated GLUT4 vesicular trafficking [89]. Furthermore, in a recent report, Rab10 has been found to play a key role in ER morphogenesis by regulating tubular extension and fusion [90]. Rab10 depletion or genetic ablation also led to an increased LD accumulation in hepatoma similar to Rab7 [91]. In starved cells, there was an interesting translocation and colocalization of Rab10 (activated) with autophagic membrane markers such as LC3 and Atg16 on the LD surface. Rab10 may enhance LD envelopment by increasing phagophore via $\mathrm{EH}$ domain-binding protein 1 and 2 (membranedeforming ATPase) functioning in a complex downstream of Rab7 during lipophagic development; it was hypothesized [91]. Although other members of the Rab family have also been associated with lipolysis and LD metabolism, however, their putative roles in LD selective autophagy are largely unknown. Colocalization of Rab32 with LD and autophagic membrane markers have also been reported in the fat body of Drosophila [92]. Furthermore, an increased ATGL level and the decrease in number and size of LDs was found upon Rab32-knockdown [93]; however, how ATGL expression was increased remains unidentified and need more indepth studies. Another member of GTPases known to contribute in LD homeostasis is Rab18. Rab18 was found tightly associated with LD surface and found to influence adiposity [94]. Upon $\beta$-adrenergic stimulation, most of this small GTPase (Rab18) was exclusively found to localize at LD surface $[95,96]$. Rab18 binding to only a subset of LDs indicates a distinct recruitment pattern that is dependent on the metabolic state of the LDs. Interactions between the proteins COP-I and TRAPP-II aid in the relocalization of TRAPPII on the surface of LDs and enhance lipolysis by activating Rab18 [97]. TRAPP complexes have been described to participate in autophagy [98]; hence, there is a need to carry out further investigations to explore any possible role of Rab18-TRAPP interactions in lipophagy. In addition to these studies, Rab25 have been recently identified in hepatic stellate cells (HSCs) to contribute in autophagy of LDs enriched in retinyl ester (Z. [99]). The increase in Rab25 expression was assumed to be caused by the release of reactive oxygen species (ROS) as a result of HSC activation. Rab25-ablation by siRNA led in LD turnover being reduced, which inhibited HSC activation. These findings point to a new role for Rab25 in lipophagy; however, it is unclear if this GTPase works just on the surface of LDs or impacts other autophagic arms.

4.3. LD-Surface Receptors in Lipophagy. Identifying the receptors involved in cargo-specific selective autophagy has been of significant interest in revealing the link between a particular organelle and autophagic machinery [9]. With the exception of LDs, these unique autophagic receptors have been identified in nearly all organelles. The majority of these receptors were discovered to interact with LC3B via LIR, as previously reported [100]. For example, the discovery of NDP52 and optineurin (two of the most essential mitochondrial cargo receptors) provided fresh and interesting insights into mitophagy [101, 102]. NDP52 may participate in other types of autophagy (i.e., xenophagy) $[103,104]$ while there are several other general candidate receptors such as p62/ SQSTM1, NBR1, and Huntingtin that play important roles in targeted turnover of several other organelles $[16,105,106]$. Furthermore, significant accumulation of LDs as a result of a mutation in Huntingtin is suggestive of a critical role of this receptor protein in LD metabolism [106, 
107]. Because LC3-binding to ATGL promotes LD degradation, no additional LD-resident proteins that unequivocally mediate lipophagy via an LC3-binding mechanism have been discovered yet. These findings point to other proteins (such as Rab7 and others) recruiting autophagic machinery via an as-yet-unidentified mechanism. Protein changes such as polyubiquitination are an alternate method for promoting lipophagy. For example, ancient ubiquitous protein 1 (AUP1) can be marked for destruction by interacting with Ube2g2 via its G2 binding domain (E2 ubiquitin conjugase G2) [108-110]. To understand the details of how this signaling is initiated and how it helps in regulating LD turnover via autophagy required more rigorous investigations on this front in the near future.

\section{Lipophagy Regulation}

5.1. Transcriptional Regulation. Over the last few decades, considerable progress has been made in understanding autophagy and, to a lesser degree, lipophagy regulation at the transcriptional level. Microphthalmia-associated/TFE subfamily of basic/helix-loop-helix/leucine zipper transcription factors, including HLH-30 (C. elegans) and TFEB and TFE3 (mammals), are the most researched autophagy/lipophagy regulators among all of these transcriptional regulators. TFEB has been discovered to enhance lipophagy as well as contribute to fat metabolism by activating PPARcoactivator 1 (PGC-1) and PPAR-targeted genes that govern fat catabolism [111]. Lipophagy and lysosomal-associated lipolysis were observed as a result of coordinated TFEB homolog HLH-30 activation that does so by enhancing the expression of many lysosomal lipases in C. elegans [50]. Moreover, starvation-induced LAL expression was found to be TFEB dependent in mice. In addition to it, TFE3 has also been found to regulate hepatic lipophagy. It is of great interest that steatosis was observed upon hepatic ablation of TFE3 while hepatic overexpression of TFE3 resulted in a decrease of steatosis via lipophagy induction [112]. TFE3 overexpression in adipocytes, on the other hand, was shown to increase obesity [113], which may be explained based on current research showing the important role of autophagy in adipocyte development $([114,115]$; C. [116]). These studies are suggestive of differential regulation of lipid metabolism by TFE3 in adipocytes and liver. Lipophagy induction and LAL expression were noted in adipose tissue as a result of starvation-induced activation of FOXO1 [41]. Furthermore, hepatic steatosis and hypertriglyceridemia were observed in liver-specific FOXO 1/3/4 knockout mice while overexpression of ATG14 resulted in a reversal of these anomalies [117]. In a similar research, cAMP response element-binding protein (CREB) was found to enhance lipophagy under deprivation by activating the TFEB, but food supplementation reversed these circumstances via farsenoid $\mathrm{X}$ receptor activation (FXR) [118]. Moreover, PPAR- $\alpha$ activation under nutrient depletion was found to inhibit FXR-mediated lipophagy suppression (J. M. [123]), and the results of this study speak about a regulatory interaction between FXR and PPAR- $\alpha$. Based on these findings, it can be concluded that starvation-induced activation of certain transcription factors and coactivators such as TFEB, TFE3, PPAR- $\alpha$, PGC- $1 \alpha$, FOXO1, and CREB is involved in promoting while nutrient supplementation-induced transcription factors, such as FXR, are involved in suppressing lipophagy. Recently, it was shown that the sterol response element-binding protein-2 (SREBP-2) is also involved in autophagy/lipophagy control. SREBP-2 knockdown activates autophagy and increases LD turnover by increasing autophagosome formation in the presence of sterol deprivation [119]. In addition to it, SREBP-2 was found to prevent liver steatosis by promoting lipophagy via enhancing the expression of PNPLA8 [67]. Therefore, based on these findings, it can be concluded that SREBP-2 compensates cholesterol deficiency not only by promoting lipophagy but also by directly increasing the biosynthesis of cholesterol by promoting the expression of the relevant genes. Furthermore, these studies throw the light on the significance of lipid signaling in lipophagy regulation.

5.2. Nutritional and Hormonal Regulation. Various mechanisms that respond to the nutrient status of cell also regulate lipophagy, such as mTOR (H. [120]), AMPK (Y. [121]), farnesoid X receptor (FXR), and peroxisome proliferatoractivated receptor- $\alpha$ (PPAR $\alpha)$ (J. M. [122]). mTOR is engaged in a variety of activities, including the control of several downstream metabolic processes. It is also known as the major signalling hub since it reacts to a variety of hormones, including insulin, as well as nutrients like glucose and amino acids. It is a strong autophagy inhibitor that is activated in response to nutrition. When rapamycin was used to block mTOR via autophagy activation, enhanced lipid oxidation and catabolism were found (mTOR inhibitor) [123]. In addition to it, LD turnover was observed to be increased in hypothalamic neurons upon autophagy induction via serum deprivation that inactivates mTOR by decreasing its phosphorylation [33]. Moreover, the interplay between the autophagic process and lipolysis via mTOR inhibition has been described in an outstanding study carried out in worms [49]. Interestingly, rapamycin was found to increase the lifespan of $C$. elegans via enhancing the activity of lysosomal lipase lipl-4. Therefore, it can be concluded that mTOR similarly regulates lipophagy and autophagy; however, how it suppresses lipophagy that remained largely unknown?

It is of great interest that hormones that are usually involved in catabolism also contribute to promoting lipophagy. For instance, $\beta$-adrenergic signaling was found to enhance the lipophagic process in adipose tissues similar to hepatocytes [85] in a Rab-7-dependent manner [84]. Thyroidine (T3) is another important hormone whose role in hepatic mitochondrial $\beta$-oxidation and LD catabolism has been well studied. Moreover, the presence of functional thyroid receptors was found to be necessary for LD turnover via autophagy (Rohit Anthony [124]). However, how lipophagy is regulated by $\mathrm{T} 3$ in extrahepatic tissues requires further more detailed investigations?

Furthermore, lipophagy activation by oleic acid is suggestive of lipophagy regulation by lipids themselves [22] This activation of lipophagy was thought to be a cellular 
response to eliminate the influx of excess lipids. Intriguingly, Kaushik et al. [33] reported similar findings in hypothalamic neurons as a result of oleic acid treatment, suggesting this response as a ubiquitous effect observed in numerous cell types [33]. On contrary to acute supplementation, chronic fat supplementation resulted in steatosis characterized by low LC3 level in LDs, and a reversal of these circumstances was observed upon hepatic ATG7 restoration (L. [34]). Furthermore, chronic fat supplementation was found to decrease lipophagy by reducing LAMP-2A that subsequently causes a reduction of PLIN2 degradation via CMA [125].

5.3. Lipophagy Regulation via Small Molecules. A vast variety of natural compounds modulate lipophagy. A polyphenolic compound, abundantly found in green tea, named epigallocatechin-3-gallate (also called EGCG) has been observed to influence hepatic autophagy/lipophagy [126, 127]. Similarly, caffeine was found to protect fatty liver disease by promoting LD catabolism via lipophagic induction [128]. Furthermore, a dietary polyphenol contained in the peel of the bergamot citrus fruit (bergamot) was shown to regulate hepatic steatosis. In the livers of rats given $50 \mathrm{mg} / \mathrm{kg}$ bergamot for three months, there was a better LD output as well as increased interactions between autophagic machinery and LDs [129]. Furthermore, other elegant studies reported the protective role of red wine bioactive resveratrol in decreasing the extent of hepatic steatosis via autophagy/lipophagy mediation (Y. [130]). In contrast to abovedescribed molecules that promote autophagy/lipophagy, tetrandrine, a bisbenzylisoquinoline alkaloid, resulted in lipid accumulation in the liver cell line by impairing the normal autophagic process. Although a range of natural compounds has been reported to modulate autophagy, however, whether these compounds also regulate lipophagy is unknown yet [131].

\section{Lipophagy in Disease States}

6.1. Lipophagy and Pathophysiology of the Liver. Lipophagy is a key regulator of LD metabolism in hepatic homeostasis; as a result, any disruption in its normal function can cause to excessive lipid buildup or steatosis, which can manifest in both alcoholic and nonalcoholic fatty liver disease (NAFLD) [132]. Furthermore, lipophagy also modulates inflammation and apoptosis, major characteristics of these ailments [133]. In the first-ever study of lipophagy, hepatic TG and cholesterol level was found to be increased in hepatocytes of mice with defective autophagy [18]. Aging, obesity, inflammation, and nonalcoholic steatohepatitis all show similar deficits defined by autophagy abnormalities (NASH). Autophagy suppression resulted in a rise in steatosis in mice given a methionine-choline diet, whereas autophagy activation resulted in a significant decrease in steatosis [134]. Autophagy activation by resveratrol was also observed to attenuate the steatosis induced by this diet, suggesting a possible therapeutic potential of this compound to treat NASH [135]. Alterations in lipids other than hydrolyze triglycerides (TGs) may influence the autophagy in fatty liver disease. For instance, an increased level of bioactive sphingolipid cer- amide was found in the liver of Atg7-ko mice [136]. Moreover, an increase in de novo sphingolipid synthesis resulted in an upregulated autophagy activity suggesting that LD catabolism via autophagy activation consume excessive LDs for liver lipid homeostasis. Similarly, ceramide was found to be upregulated in several other ailments including diabetes [137] and obesity [138], both of which are associated with steatosis development [139]; therefore, it can be speculated that autophagy defect may be a leading cause of pathologies related to altered sphingolipid contents. In agreement with these investigations, knockdown of Atg14 ([117]) or Tfeb-ko [111] resulted in the enhancement of hepatic TG level. Moreover, autophagy enhancement in obese mice via Atg7/Atg14 overexpression alleviated the steatosis extent $[34,91]$. Although there is a huge number of studies in favor of alleviation of hepatic LD accumulation by lipophagy, however, some mouse models showed no or less steatosis upon autophagic defect [140, 141]. This incongruity may be a result of differences in experimental setups and animal models. It was suggested that lipophagy perhaps replenish LDs with fatty acids (FAs) during starvation in vitro, while lipids are removed from LDs by lipolysis [142]. However, this study was conducted on fibroblasts with low lipid content, which were not grown in a serumfree medium but rather in a saline solution for a longer period of time. As a result, study's findings are unworthy of consideration and remain unclear. As a result, in this circumstance, more comprehensive in vivo studies are needed to determine whether lipophagy reduces hepatic damage and steatosis in NAFLD patients. In acute ethanol-induced in vitro and in vivo models of liver steatosis and damage, LD degradation was revealed to be mediated via autophagy [143]. ROS intermediates, produced as a result of acute alcohol consumption, were found to induce lipophagy [143]. Similar observations were found in mice fed on ethanol diet or upon overexpression of CYP2E1 (prooxidant enzyme) ([144]). Furthermore, a lower level of the main antioxidant glutathione in the liver of mice given an alcoholic diet, which was further reduced when autophagy was inhibited, clearly implies that autophagy plays a protective role against chronic alcohol-induced damage. On the basis of the aforementioned, it can be inferred that LD degradation via autophagy protects against ethanol-induced liver damage and oxidative stress in both acute and chronic forms. This protective role may be a result of excess lipid removal that acts as a substrate for oxidative stress or via ATP supplementation generated as a result of free fatty acids (FFAs) catabolism via lipophagy, that is, the possible mechanism of resistance adopted by hepatic cells against oxidative stress [145]. ATGL and hormone-sensitive lipase (HSL) are the major factors responsible for the hydrolysis of triglycerides in adipocytes. Though ATGL shows highest expression in fat tissues, a lower level of activity is also found in several nonadipose tissues. ATGL deficiency led to an enhanced LD accumulation in liver and heart besides adipose tissues in mice. However, ATGL is involved in varying degrees of phospholipid hydrolysis while no hydrolysis of cholesteryl esters was observed under various experimental conditions. Furthermore, HSL is expressed normally at an extremely 
low level in liver, and there are no studies so far describing an association between HSL and lipolysis in the liver. On the other hand, a reversal of hepatic steatosis was observed upon HSL overexpression ex vivo [146]. "Two-hit theory" is the age-old and reliable model for the study of development and progression of NASH ([147]; [148]). The first strike is defined by the onset of fatty liver disease, followed by the second hit, which is characterised by the accumulation of reactive oxygen species (ROS), liver inflammation, and mitochondrial dysfunction, all of which lead to NASH. However, the exact mechanism by which autophagy mediates the two-hit scenario for NASH development is yet unknown. Lipophagy was initially discovered to prevent excessive fat accumulation during the first hit [22]. Secondly, autophagy helps in the elimination of ROS-producing mitochondria and maintaining ATP levels preventing the second hit. TNF- $\alpha$ is an inflammatory molecule that mediates hepatic injury, and it has been reported that Atg-7 deficiency led to hepatic injury upon administration of common hepatic toxicants including galactosamine combined with LPS/galactosamine with TNF- $\alpha$ to mice [149]. Lipophagy plays an important role in protecting hepatocytes against lipotoxicity. SIRT3, a $\mathrm{NAD}^{+}$-dependent deacetylase, promotes macroautophagy through AMPK-dependent pathway, downregulates SCD1 to inhibit lipogenesis, and activates LCAD to promote $\beta$-oxidation of fatty acids and in this way mitigate lipotoxicity ([150]). Impaired lipophagy is associated with advancement in NAFLD [151]. Recently, another study conducted by [152] have reported that dietary intake and exercise help in controlling NAFLD by enhancing lipophagy. These therapies enhance lipophagy in liver by downregulating Akt/mTOR/ULK1 pathways and upregulating AMPK/ULK1 pathways ([152]). Moreover, lipophagy not only prevents hepatic lipotoxicity but also helps in VLDL production by providing free fatty acids. In FFAdeficient conditions, nearly $70 \%$ of secreted VLDL comes from the TG degradation in hepatocytes; however, mechanism of TG breakdown to make VLDL remains unclear [153]. Moreover, autophagy inhibition resulted in reduced fatty acid oxidation and VLDL production, and autophagy activation led to an enhanced VLDL production [154, 155]. In human subjects, autophagy examination has always remained a major restraint to truly find the extent of autophagy defect in human NAFLD. In addition to it, the samples need to be pretreated with lysosomal inhibitors to measure autophagy flux and activity [156, 157]. Despite these constraints, correlative studies have provided evidence of the contribution of autophagy in the prevention of NAFLD development in humans. For instance, autophagy cargo p62/SQSTM1 was found to be accumulated in liver sections from expired subjects and in biopsy samples obtained from subjects with severe steatosis [158, 159]. As a result, an inverse relationship between autophagy and liver total lipid concentration may be hypothesised. Furthermore, autophagy inhibition by pharmaceutical drugs used to treat steatosis reinforces the link between steatosis and lipophagic activity. For example, thymidine analogues (stavudine and zidovudine), which are frequently used as antiretroviral treatment, have been shown to reduce autophagy and may be responsible for the elevated LD content in these patients' livers [160]. On the contrary, steatosis development was prevented by anticonvulsant carbamazepine which is capable of activating autophagy. Recently, obesity and related lipotoxic states have been found to inhibit autophagy perhaps due to an increased cytosolic calcium level [161]. Moreover, this calcium led to an impairment of autophagosome-lysosome fusion [161]. To further dig out this finding, obese mice were administered calcium channel blocker verapamil that resulted in autophagy activation and decreased the LD accumulation and in turn improved insulin sensitivity [161]. Collectively, these investigations from humans and mice models speak about a major function of lipophagy in LD homeostasis in liver and formulate the basis to fully understand the molecular mechanisms responsible for lipid metabolism mediated by lipophagy (autophagy) [162].

6.2. Lipophagy in Stellate Cell Activation and Fibrosis. Lipophagy transforms stellate cells into matrix-producing myofibroblasts, resulting in fibrosis as a pathologic condition. In their dormant condition, these stellate cells tend to accumulate more lipids, mostly in the form of vitamin A. During the activation of stellate cells, these lipid reserves are digested. In hepatic stellate cells, fibrotic stimuli were observed to increase macroautophagy [163, 164]. Autophagy-defective stellate cells were failed to activate, and restoration of activation potential upon FFA oleate supplementation is suggestive of lipophagy-driven transdifferentiation upon substrate supplementation to obtain energy required for their activation [163]. Moreover, a decrease in fibrotic extent was observed in vivo in autophagy-deficient stellate cells [163]. Whether lipophagy is responsible for fibrosis in organs other than the liver is still unknown; however, the prime importance of stellate cell activation in fibrosis development and progression is suggestive of significant roles of lipophagy in this cell that may be critical in some fibrotic disorders. Taken into account the previously described role of lipophagy in liver steatosis, fibrosis, and injury, it can be suggested that autophagy may influence multiple hepatic features of NAFLD affecting a variety of cells [165].

6.3. Lipophagy in Metabolic Disorders. The finding that autophagy functions in adipogenesis have thrown in gear a considerable interest in investigating whether any possible association exists between autophagy and metabolic disorders, i.e., obesity. A direct correlation was found between autophagy level and fat depot size among human subjects with varying degrees and types of obesity. Intriguingly, significantly high autophagic activity was observed in omental fat tissue samples from obese subjects, and as a matter of fact, a further increase in autophagy extent was found in obese individuals with insulin resistance [166]. In addition to the functional involvement of autophagy in the differentiation of adipose tissues during development, our findings imply that autophagy may play a role in controlling the size of adipose tissues and lipid homeostasis in adulthood. The increase of autophagy prior to obesity-related morbidity suggests that autophagy activation may be used as a defence mechanism to deal with excessive cellular lipid levels. 
Autophagy's effects and outcomes, on the other hand, might vary depending on metabolic status. For example, mTOR attenuation has recently been reported in adipocytes from type 2 diabetes patients, and this discovery lends credence to the idea that this might be the mechanism behind autophagy increase in these cells. As a result of the accelerated production of LDs and their increased autophagic activity, excessive FFA is released from LDs, posing cellular toxicity. In these cases, autophagy blockage or autophagy decrease may be a preferable technique [167]. Lipophagy has been put forward recently as a probable defensive strategy to prevent atherosclerosis resulting from atypical lipid amassing in macrophage foam cells [168]. Furthermore, lipolytic LD mobilization in foam cells mediated by autophagy was also proposed as a potential mechanism responsible for atherogenesis. Lipid loading resulted in an increased macrophage lipophagy both in vitro and in vivo, and an abnormal cholesterol removal was observed upon autophagy defect in mice [42]. It can be speculated based on these findings that higher concentrations of these circulating lipids may impair the autophagic activity of macrophages and resulting in intracytoplasmic lipid deposits transform them into "foam cells" [35]. The subsequent lipid deposition is the seeding to favor atherosclerotic plaque development. Therefore, present therapeutic attempts are targeting macrophages to reduce the extent of lipid buildup in the form of endothelial plaques by favoring the cholesterol efflux from them. Consequently, interventions aimed at promoting autophagy and enhancing cholesterol efflux are promising therapeutic tools in the cure of atherosclerosis. Interestingly, the potential of arterial smooth muscle cells may also be utilized in the manipulation for autophagy upregulation to reduce arterial plaque formation. Furthermore, the inflammation associated with plaque formation is a consequence of the compromised autophagic activity of these cells [169].

6.4. Lipophagy in Aging and Longevity. The autophagy process was found to be declined in many of the tissues and organisms as the aging process goes on [170]. This decrease in autophagy, particularly lipophagy, resulted in the buildup of LDs, and the resulting large lipid depots exacerbated the situation by further impeding the autophagic process, favouring a path for the metabolic syndrome of ageing via a potential feedback loop. Massive lipid accumulation in organs, hypercholesterolemia, and insulin resistance are all symptoms of the metabolic syndrome of ageing. Antilipolytic medicines have demonstrated the ability to ameliorate not just hypercholesterolemic conditions but also general health span in mice models of ageing, which is of considerable interest [171]. However, their beneficial effects are thought to be a result of autophagy induction in response to increasing cytoplasmic lipid contents [172, 173]. Genetic associations among autophagic process, longevity, and atherogenesis have been found recently in C. elegans [49]. Functional autophagy was found to activate LAL-4, and in turn, this lipase showed a potential of autophagy induction. Interestingly, the autophagic process and lipase need to work together for lifespan extension upon germline elimination. Though the particular target and how it affects autophagy

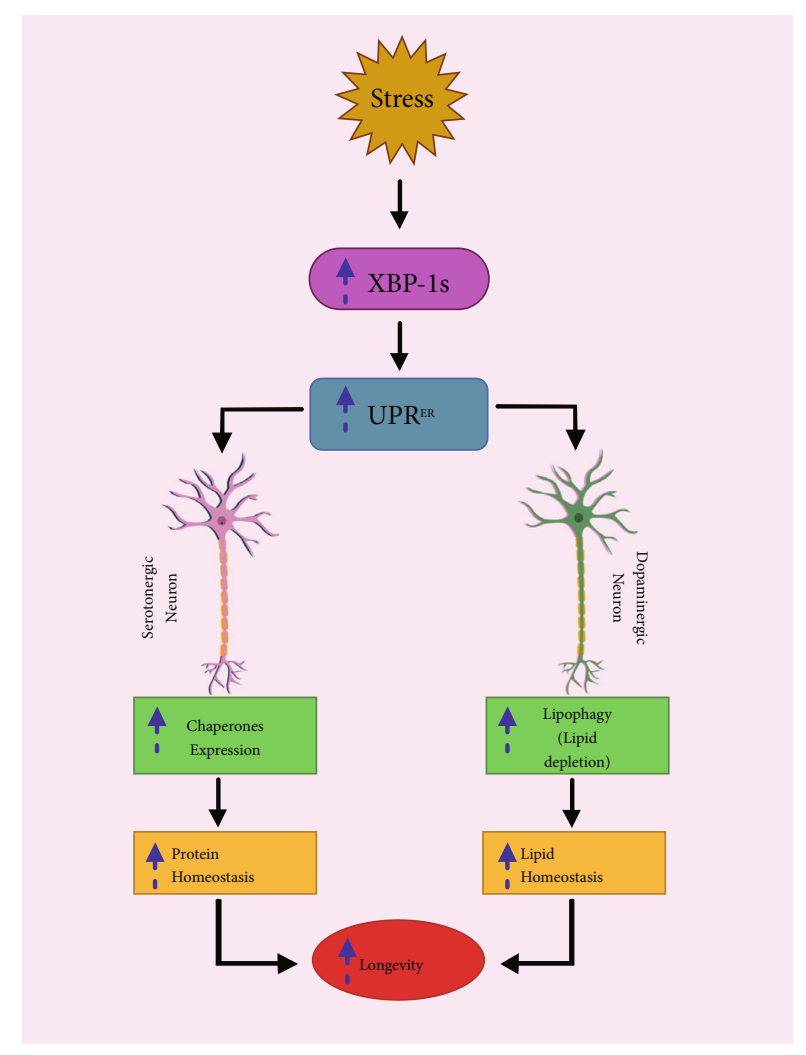

FIGURE 3: XBP-1s enhances longevity by promoting lipid and protein homeostasis via two independent pathways mediated by $\mathrm{UPR}^{\mathrm{ER}}$. XBP-1 induction promotes protein homeostasis via chaperones and lipid homeostasis via restructuring ER. This restructuring enhances lipophagic depletion of LDs.

remains unknown, it can be speculated that it contributes in longevity and lifespan extension by better handling of cytoplasmic lipid contents via lipophagy [174]. Recent studies have found that unfolded protein response of the endoplasmic reticulum (UPR ${ }^{\mathrm{ER}}$ ) extend lifespan in an $x b p-1 s$ dependent manner [175] which is unregulated in response to stress [176]. $\mathrm{UPR}^{\mathrm{ER}}$ reverses onset of aging and extends life span by two independent pathways. UPR ${ }^{\mathrm{ER}}$ regulates protein homeostasis via upregulating the expression of chaperones; on the other hand, it enhances depletion of lipid droplets by promoting lipophagy via restructuring ER morphology [177, 178]. Moreover, it has been found that dopaminergic neurons activate $\mathrm{UPR}^{\mathrm{ER}}$ to drive lipid homeostasis, and serotonergic neurons drive protein homeostasis by upregulating $\mathrm{UPR}^{\mathrm{ER}}$. These two events in turn enhance longevity (Figure 3)

6.5. Lipophagy and Viral Replication. Removal of superfluous cellular elements by autophagy is suggestive of its involvement in the elimination of intracellular pathogens [172]. In contrary to popular belief, certain infectious agents may be able to manipulate this degradative route to their advantage. Hepatitis viruses are the most common cause of chronic hepatitis, and they modify autophagy in host cells by an unknown mechanism. HCV causes an increase in autophagy $[179,180]$ and $\operatorname{HBV}[181,182]$, and this 


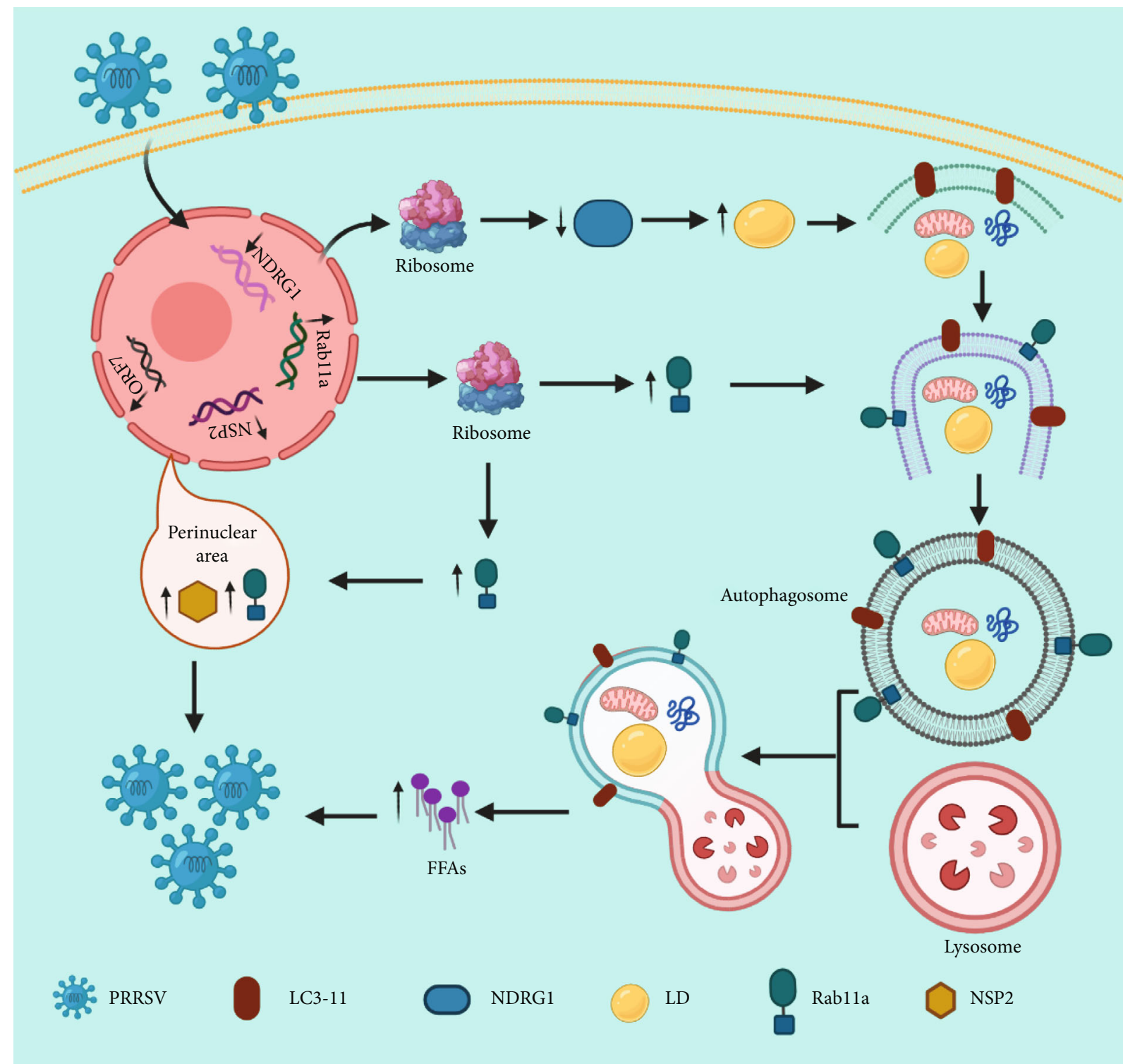

FIGURE 4: PRRSV infection promotes downregulation of NDRG1 and upregulation of Rab11a to promote replication: viral infection reduces the expression of NDRG1 and enhances expression of Rab1la, both in turn promote lipophagy by inducing autophagy and enhancing autophagosomes maturation respectively. Lipophagy promote viral replication by providing FFAs via LD degradation.

augmentation of autophagy helps in the replication of viruses instead of their elimination. An association exists between the HCV core proteins and LDs that helps in viral assembly via an unknown mechanism [183]. Similarly, autophagy augmentation has also found to enhance the replication of the dengue virus via the lipophagic mechanism. LD-associated replication, similar to $\mathrm{HCV}$, has also been found in the dengue virus; however, the site of assembly remains ambiguous [184]. It has been recently described that autophagy may provide the energy required for viral replication by metabolizing the lipids [185]. In hepatoma cells, the dengue virus supported LD-coupled autophagosome development. In infected hepatoma cells, lipophagy resulted in an increase in -oxidation and a significant drop in LD volume and TG. Furthermore, inhibiting autophagy caused an increase in -oxidation levels, which halted viral propagation. When FFA was added, the replication barrier was removed, indicating that autophagic breakdown of LDs resulted in the release of FFAs that were metabolised by -oxidation to enhance viral replication. Replication of HCV has also been shown to be dependent on $\beta$-oxidation [185]. In hepatocytes, induction of lipophagy by these viruses is a key event in the viral life cycle and may serve as a potential novel therapeutic target for future therapies. The exact mechanism involved in viral induction is still unknown; however, NS4A has been identified as a potential candidate involved in its induction [186]. Autophagy was found to restrict the replication of dengue virus in monocytes; therefore, it can be speculated that autophagy induced by viruses may vary in other types of cells [187]. Moreover, further investigations are required to explore whether lipophagy is involved in the replication of the viruses that alter 
the autophagic function such as HBV (K. [188]). According to Wang et al. [189], PRRSV infection increases the expression of Rab1la at both the mRNA and protein levels. Increased Rab1la expression promotes autophagosome maturation, which leads to lipophagy. NSP2 and ORF7 expression was decreased when Rab1la expression was disrupted. Rab1la silencing resulted in an accumulation of LC3-II and p62, indicating Rab11a's function in autophagy surge. Furthermore, NSP2 and Rab11a were discovered to be colocalized, indicating that Rab11a aids viral replication by functioning as a proviral host factor (K. [189]). Recently, it was found that downregulation of NDRG1 expression promote PRRSV (Porcine reproductive and respiratory syndrome) replication by promoting lipophagy which in turn increase the level of FFAs by increasing LD degradation (Figure 4) ([190]).

\section{Conclusion}

Considering the facts described above, it can be inferred that autophagy is engaged in the metabolism of a range of substances, with cytosolic lipids lately being added to the list of targets. In the last decade, research on the function of autophagy in lipid metabolism has advanced our understanding of how autophagy uses lysosomes to maintain lipid homeostasis. The specific molecular processes of most of the major activities involved in LD trafficking and catabolism, as mentioned above, are, nevertheless, largely unknown. Despite several studies, many unsolved issues remain, including (1) how lipids are selectively directed towards lysosomes? What are their molecular routes, exactly? (2) How can lysosomes distinguish between different types of lipids? (3) How does lipophagy break down particular lipid species? (4) How is the equilibrium between various lipid catabolic pathways maintained? (5) How can the integrity and cross-talk between selective and nonselective LD breakdown through autophagy be maintained? Furthermore, because the lysosome is the primary destination of autophagy and other endocytic processes, identifying particular proteins that regulate lipophagy might have therapeutic implications. Because autophagy (lipophagy) dysfunction is linked to a range of pathological disease states, additional in-depth research to uncover the specific molecular processes is recommended.

\section{Data Availability}

There is no additional supporting data in this article.

\section{Conflicts of Interest}

All the authors declared there is no conflict of interest or ethical concern in this article.

\section{Authors' Contributions}

MBK, MHA, and MR collected the data, draw figures, and wrote the manuscript; RM and $\mathrm{NN}$ modified the figures and help out in improving the manuscript; MBK, MHA, and NS proposed the idea, supervised, and approved the final version of the manuscript. Muhammad Babar Khawar, Muddasir Hassan Abbasi, and Mussarat Rafiq contributed equally to this work

\section{Acknowledgments}

The authors are thankful to the vice-chancellors of the University of the Punjab, Lahore-Pakistan, and University of Okara, Okara, Pakistan, for providing support for the accomplishment of this study. Figures 3 and 4 were designed using BioRender platform.

\section{References}

[1] C. A. Lamb, T. Yoshimori, and S. A. Tooze, "The autophagosome: origins unknown, biogenesis complex," Nature Reviews Molecular Cell Biology, vol. 14, no. 12, pp. 759774, 2013.

[2] N. Mizushima and M. Komatsu, "Autophagy: renovation of cells and tissues," Cell, vol. 147, no. 4, pp. 728-741, 2011.

[3] N. Mizushima, T. Yoshimori, and Y. Ohsumi, "The role of Atg proteins in autophagosome formation," Annual Review of Cell and Developmental Biology, vol. 27, no. 1, pp. 107132, 2011.

[4] N. Mizushima and B. Levine, "Autophagy in mammalian development and differentiation," Nature Cell Biology, vol. 12, no. 9, pp. 823-830, 2010.

[5] J. L. Schneider and A. M. Cuervo, "Autophagy and human disease: emerging themes," Current Opinion in Genetics \& Development, vol. 26, pp. 16-23, 2014.

[6] H. Gao, M. B. Khawar, and W. Li, "Autophagy in reproduction," Autophagy: Biology and Diseases, vol. 1206, pp. 453468, 2019.

[7] R. Mackeh, D. Perdiz, S. Lorin, P. Codogno, and C. Pous, "Autophagy and microtubules-new story, old players," Journal of Cell Science, vol. 126, no. 5, pp. 1071-1080, 2013.

[8] K. Suzuki and Y. Ohsumi, "Current knowledge of the preautophagosomal structure (PAS)," FEBS Letters, vol. 584, no. 7, pp. 1280-1286, 2010.

[9] V. Rogov, V. Dotsch, T. Johansen, and V. Kirkin, "Interactions between autophagy receptors and ubiquitin-like proteins form the molecular basis for selective autophagy," Molecular Cell, vol. 53, no. 2, pp. 167-178, 2014.

[10] A. Stolz, A. Ernst, and I. Dikic, "Cargo recognition and trafficking in selective autophagy," Nature Cell Biology, vol. 16, no. 6, pp. 495-501, 2014.

[11] K. Lu, I. Psakhye, and S. Jentsch, "Autophagic clearance of polyQ proteins mediated by ubiquitin-Atg8 adaptors of the conserved CUET protein family," Cell, vol. 158, no. 3, pp. 549-563, 2014.

[12] K. Okamoto, "Organellophagy: eliminating cellular building blocks via selective autophagy," Journal of Cell Biology, vol. 205, no. 4, pp. 435-445, 2014.

[13] R. J. Youle and D. P. Narendra, "Mechanisms of mitophagy," Nature Reviews Molecular Cell Biology, vol. 12, no. 1, pp. 914, 2011.

[14] J. C. Farre, R. Manjithaya, R. D. Mathewson, and S. Subramani, "PpAtg30 tags peroxisomes for turnover by selective autophagy," Developmental Cell, vol. 14, no. 3, pp. 365-376, 2008. 
[15] A. M. Motley, J. M. Nuttall, and E. H. Hettema, "Pex3anchored Atg36 tags peroxisomes for degradation inSaccharomyces cerevisiae," The EMBO Journal, vol. 31, no. 13, pp. 2852-2868, 2012.

[16] E. Deosaran, K. B. Larsen, R. Hua et al., "NBR1 acts as an autophagy receptor for peroxisomes," Journal of Cell Science, vol. 126, article 114819, 2012.

[17] P. K. Kim, D. W. Hailey, R. T. Mullen, and J. LippincottSchwartz, "Ubiquitin signals autophagic degradation of cytosolic proteins and peroxisomes," Proceedings of the National Academy of Sciences, vol. 105, no. 52, pp. 20567-20574, 2008.

[18] S. Schuck, C. M. Gallagher, and P. Walter, "ER-phagy mediates selective degradation of endoplasmic reticulum independently of the core autophagy machinery," Journal of Cell Science, vol. 127, article 154716, 2014.

[19] W. E. Dowdle, B. Nyfeler, J. Nagel et al., "Selective VPS34 inhibitor blocks autophagy and uncovers a role for NCOA4 in ferritin degradation and iron homeostasis in vivo," Nature Cell Biology, vol. 16, no. 11, pp. 1069-1079, 2014.

[20] J. D. Mancias, X. Wang, S. P. Gygi, J. W. Harper, and A. C. Kimmelman, "Quantitative proteomics identifies NCOA4 as the cargo receptor mediating ferritinophagy," Nature, vol. 509, no. 7498, pp. 105-109, 2014.

[21] A. Schreiber and M. Peter, "Substrate recognition in selective autophagy and the ubiquitin-proteasome system," Biochimica et Biophysica Acta (BBA)-Molecular Cell Research, vol. 1843, no. 1, pp. 163-181, 2014.

[22] R. Singh, S. Kaushik, Y. Wang et al., "Autophagy regulates lipid metabolism," Nature, vol. 458, no. 7242, pp. 1131$1135,2009$.

[23] E. J. Bonten, I. Annunziata, and A. d'Azzo, "Lysosomal multienzyme complex: pros and cons of working together," Cellular and Molecular Life Sciences, vol. 71, no. 11, pp. 2017-2032, 2014.

[24] F. Wilfling, J. T. Haas, T. C. Walther, and R. V. F. Jr, "Lipid droplet biogenesis," Current Opinion in Cell Biology, vol. 29, pp. 39-45, 2014.

[25] V. K. Khor, W. J. Shen, and F. B. Kraemer, "Lipid droplet metabolism," Current Opinion in Clinical Nutrition and Metabolic Care, vol. 16, no. 6, pp. 632-637, 2013.

[26] A. R. Kimmel, D. L. Brasaemle, M. McAndrews-Hill, C. Sztalryd, and C. Londos, "Adoption of PERILIPIN as a unifying nomenclature for the mammalian PAT-family of intracellular lipid storage droplet proteins," Journal of Lipid Research, vol. 51, no. 3, pp. 468-471, 2010.

[27] A. Filipe and J. McLauchlan, "Hepatitis C virus and lipid droplets: finding a niche," Trends in Molecular Medicine, vol. 21, no. 1, pp. 34-42, 2015.

[28] N. B. Cole, D. D. Murphy, T. Grider, S. Rueter, D. Brasaemle, and R. L. Nussbaum, "Lipid Droplet Binding and Oligomerization Properties of the Parkinson's Disease Protein $\alpha$-Synuclein," Journal of Biological Chemistry, vol. 277, no. 8, pp. 6344-6352, 2002.

[29] K. A. D. Mattos, E. N. Sarno, M. C. V. Pessolani, and P. T. Bozza, "Deciphering the contribution of lipid droplets in leprosy: multifunctional organelles with roles in Mycobacterium leprae pathogenesis," Memórias do Instituto Oswaldo Cruz, vol. 107, suppl 1, pp. 156-166, 2012.

[30] A. S. Greenberg, R. A. Coleman, F. B. Kraemer et al., "The role of lipid droplets in metabolic disease in rodents and humans," The Journal of Clinical Investigation, vol. 121, no. 6, pp. 2102-2110, 2011.

[31] H. Yang, A. Galea, V. Sytnyk, and M. Crossley, "Controlling the size of lipid droplets: lipid and protein factors," Current Opinion in Cell Biology, vol. 24, no. 4, pp. 509-516, 2012.

[32] S. C. Souza, K. V. Muliro, L. Liscum et al., "Modulation of Hormone-sensitive Lipase and Protein Kinase A-mediated Lipolysis by Perilipin A in an Adenoviral Reconstituted System," Journal of Biological Chemistry, vol. 277, no. 10, pp. 8267-8272, 2002.

[33] S. Kaushik, J. A. Rodriguez-Navarro, E. Arias et al., "Autophagy in hypothalamic AgRP neurons regulates food intake and energy balance," Cell Metabolism, vol. 14, no. 2, pp. 173-183, 2011.

[34] L. Yang, P. Li, S. Fu, E. S. Calay, and G. S. Hotamisligil, "Defective hepatic autophagy in obesity promotes ER stress and causes insulin resistance," Cell Metabolism, vol. 11, no. 6, pp. 467-478, 2010.

[35] H. Koga, S. Kaushik, and A. M. Cuervo, "Altered lipid content inhibits autophagic vesicular fusion," The FASEB Journal, vol. 24, no. 8, pp. 3052-3065, 2010.

[36] H. I. Cho, J. W. Choi, and S. M. Lee, "Impairment of autophagosome-lysosome fusion contributes to chronic ethanol- induced liver injury," Alcohol, vol. 48, no. 7, pp. 717-725, 2014.

[37] H. Koga, S. Kaushik, and A. M. Cuervo, "Inhibitory effect of intracellular lipid load on macroautophagy," Autophagy, vol. 6, no. 6, pp. 825-827, 2010.

[38] X. Xu, A. Grijalva, A. Skowronski, M. van Eijk, M. J. Serlie, and A. W. Ferrante, "Obesity activates a program of lysosomal-dependent lipid metabolism in adipose tissue macrophages independently of classic activation," Cell Metabolism, vol. 18, no. 6, pp. 816-830, 2013.

[39] R. R. Kaini, L. O. Sillerud, S. Zhaorigetu, and C. A. A. Hu, "Autophagy regulates lipolysis and cell survival through lipid droplet degradation in androgen-sensitive prostate cancer cells," The Prostate, vol. 72, no. 13, pp. 1412-1422, 2012.

[40] V. M. Hubbard, R. Valdor, B. Patel, R. Singh, A. M. Cuervo, and F. Macian, "Macroautophagy regulates energy metabolism during effector T cell activation," The Journal of Immunology, vol. 185, no. 12, pp. 7349-7357, 2010.

[41] D. Lettieri Barbato, G. Tatulli, K. Aquilano, and M. R. Ciriolo, "FoxO1 controls lysosomal acid lipase in adipocytes: implication of lipophagy during nutrient restriction and metformin treatment," Cell Death \& Disease, vol. 4, no. 10, article e861, 2013.

[42] M. Ouimet, V. Franklin, E. Mak, X. Liao, I. Tabas, and Y. L. Marcel, "Autophagy regulates cholesterol efflux from macrophage foam cells via lysosomal acid lipase," Cell metabolism, vol. 13, no. 6, pp. 655-667, 2011.

[43] M. Ouimet and Y. L. Marcel, "Regulation of lipid droplet cholesterol efflux from macrophage foam cells," Arteriosclerosis, Thrombosis, and Vascular Biology, vol. 32, no. 3, pp. 575$581,2012$.

[44] M. Martinez-Vicente, Z. Talloczy, E. Wong et al., "Cargo recognition failure is responsible for inefficient autophagy in Huntington's disease," Nature Neuroscience, vol. 13, no. 5, pp. 567-576, 2010.

[45] S. A. Khaldoun, M. A. Emond-Boisjoly, D. Chateau et al., "Autophagosomes contribute to intracellular lipid 
distribution in enterocytes," Molecular biology of the cell, vol. 25, no. 1, pp. 118-132, 2014.

[46] T. van Zutphen, V. Todde, R. de Boer et al., "Lipid droplet autophagy in the yeastSaccharomyces cerevisiae," Molecular Biology of the Cell, vol. 25, no. 2, pp. 290-301, 2014.

[47] C. W. Wang, Y. H. Miao, and Y. S. Chang, "A sterol-enriched vacuolar microdomain mediates stationary phase lipophagy in budding yeast," Journal of Cell Biology, vol. 206, no. 3, pp. 357-366, 2014.

[48] L. N. Nguyen, J. Bormann, G. T. T. le et al., "Autophagyrelated lipase FgATG15 of Fusarium graminearum is important for lipid turnover and plant infection," Fungal Genetics and Biology, vol. 48, no. 3, pp. 217-224, 2011.

[49] L. R. Lapierre, S. Gelino, A. Melendez, and M. Hansen, "Autophagy and lipid metabolism coordinately modulate life span in germline-less C. elegans," Current Biology, vol. 21, no. 18, pp. 1507-1514, 2011.

[50] E. J. O'Rourke and G. Ruvkun, "MXL-3 and HLH-30 transcriptionally link lipolysis and autophagy to nutrient availability," Nature Cell Biology, vol. 15, no. 6, p. 668, 2013.

[51] T. Kurusu, T. Koyano, S. Hanamata et al., "OsATG7 is required for autophagy-dependent lipid metabolism in rice postmeiotic anther development," Autophagy, vol. 10, no. 5, pp. 878-888, 2014.

[52] M. Oku, Y. Takano, and Y. Sakai, "The emerging role of autophagy in peroxisome dynamics and lipid metabolism of phyllosphere microorganisms," Frontiers in Plant Science, vol. 5, p. 81, 2014.

[53] E. Itakura, C. Kishi-Itakura, and N. Mizushima, "The hairpin-type tail-anchored SNARE syntaxin 17 targets to autophagosomes for fusion with endosomes/lysosomes," Cell, vol. 151, no. 6, pp. 1256-1269, 2012.

[54] U. Nair, A. Jotwani, J. Geng et al., "SNARE proteins are required for macroautophagy," Cell, vol. 146, no. 2, pp. 290-302, 2011.

[55] R. J. Schulze, S. G. Weller, B. Schroeder et al., "Lipid droplet breakdown requires dynamin 2 for vesiculation of autolysosomal tubules in hepatocytes," Journal of Cell Biology, vol. 203, no. 2, pp. 315-326, 2013.

[56] Y. Liu, Y. Takahashi, N. Desai et al., "Bif-1 deficiency impairs lipid homeostasis and causes obesity accompanied by insulin resistance," Scientific Reports, vol. 6, no. 1, p. 20453, 2016.

[57] G. Onal, O. Kutlu, D. Gozuacik, and S. Dokmeci Emre, "Lipid droplets in health and disease," Lipids in Health and Disease, vol. 16, no. 1, p. 128, 2017.

[58] A. R. Kimmel and C. Sztalryd, "The perilipins: major cytosolic lipid droplet-associated proteins and their roles in cellular lipid storage, mobilization, and systemic homeostasis," Annual Review of Nutrition, vol. 36, pp. 471-509, 2016.

[59] S. Kaushik and A. M. Cuervo, "Degradation of lipid dropletassociated proteins by chaperone-mediated autophagy facilitates lipolysis," Nature Cell Biology, vol. 17, no. 6, p. 759, 2015.

[60] S. Kaushik and A. M. Cuervo, "AMPK-dependent phosphorylation of lipid droplet protein PLIN2 triggers its degradation by CMA," Autophagy, vol. 12, no. 2, pp. 432-438, 2016.

[61] N. Martinez-Lopez, M. Garcia-Macia, S. Sahu et al., "Autophagy in the CNS and periphery coordinate lipophagy and lipolysis in the brown adipose tissue and liver," Cell Metabolism, vol. 23, no. 1, pp. 113-127, 2016.
[62] A. Sathyanarayan, M. T. Mashek, and D. G. Mashek, "ATGL promotes autophagy/lipophagy via SIRT1 to control hepatic lipid droplet catabolism," Cell Reports, vol. 19, no. 1, pp. 19, 2017.

[63] S. A. Khan, A. Sathyanarayan, M. T. Mashek, K. T. Ong, E. E. Wollaston-Hayden, and D. G. Mashek, "ATGL-catalyzed lipolysis regulates SIRT1 to control PGC-1 alpha/PPARalpha signaling," Diabetes, vol. 64, no. 2, pp. 418-426, 2015.

[64] I. H. Lee, L. Cao, R. Mostoslavsky et al., "A role for the NADdependent deacetylase Sirt1 in the regulation of autophagy," Proceedings of the National Academy of Sciences, vol. 105, no. 9, pp. 3374-3379, 2008.

[65] M. B. Khawar, C. Liu, F. Gao et al., "Sirt1 regulates testosterone biosynthesis in Leydig cells via modulating autophagy," Protein \& Cell, vol. 12, no. 1, pp. 67-75, 2020.

[66] N. Dupont, S. Chauhan, J. Arko-Mensah et al., "Neutral lipid stores and lipase PNPLA5 contribute to autophagosome biogenesis," Current Biology, vol. 24, no. 6, pp. 609-620, 2014.

[67] K. Y. Kim, H. J. Jang, Y. R. Yang et al., "SREBP-2/PNPLA8 axis improves non-alcoholic fatty liver disease through activation of autophagy," Scientific Reports, vol. 6, no. 1, article 35732, 2016.

[68] M. Goeritzer, N. Vujic, S. Schlager et al., "Active autophagy but not lipophagy in macrophages with defective lipolysis," Biochimica et Biophysica Acta (BBA)-Molecular and Cell Biology of Lipids, vol. 1851, no. 10, pp. 1304-1316, 2015.

[69] S. Cermelli, Y. Guo, S. P. Gross, and M. A. Welte, "The lipiddroplet proteome reveals that droplets are a protein-storage depot," Current Biology, vol. 16, no. 18, pp. 1783-1795, 2006.

[70] S. A. Khan, E. E. Wollaston-Hayden, T. W. Markowski, L. Higgins, and D. G. Mashek, "Quantitative analysis of the murine lipid droplet-associated proteome during dietinduced hepatic steatosis," Journal of Lipid Research, vol. 56, no. 12, pp. 2260-2272, 2015.

[71] L. Li, H. Zhang, W. Wang et al., "Comparative proteomics reveals abnormal binding of ATGL and dysferlin on lipid droplets from pressure overload-induced dysfunctional rat hearts," Scientific Reports, vol. 6, no. 1, article 19782, 2016.

[72] P. Liu, Y. Ying, Y. Zhao, D. I. Mundy, M. Zhu, and R. G. Anderson, "Chinese hamster ovary K2 cell lipid droplets appear to be metabolic organelles involved in membrane traffic," Journal of Biological Chemistry, vol. 279, no. 5, pp. 37873792, 2004.

[73] H. Stenmark, "Rab GTPases as coordinators of vesicle traffic," Nature reviews Molecular cell biology, vol. 10, no. 8, p. 513, 2009.

[74] R. S. Kiss and T. Nilsson, "Rab proteins implicated in lipid storage and mobilization," Journal of Biomedical Research, vol. 28, no. 3, p. 169, 2014.

[75] M. G. Gutierrez, D. B. Munafo, W. Beron, and M. I. Colombo, "Rab7 is required for the normal progression of the autophagic pathway in mammalian cells," Journal of Cell Science, vol. 117, no. 13, pp. 2687-2697, 2004.

[76] S. Jager, C. Bucci, I. Tanida et al., "Role for Rab7 in maturation of late autophagic vacuoles," Journal of Cell Science, vol. 117, no. 20, pp. 4837-4848, 2004.

[77] R. Vitelli, M. Santillo, D. Lattero et al., "Role of the small GTPase Rab7 in the late endocytic pathway," Journal of Biological Chemistry, vol. 272, no. 7, pp. 4391-4397, 1997.

[78] N. Furuta, N. Fujita, T. Noda, T. Yoshimori, and A. Amano, "Combinational soluble N-ethylmaleimide-sensitive factor 
attachment protein receptor proteins VAMP8 and Vtilb mediate fusion of antimicrobial and canonical autophagosomes with lysosomes," Molecular Biology of the Cell, vol. 21, no. 6, pp. 1001-1010, 2010.

[79] H. J. . Balderhaar and C. Ungermann, "CORVET and HOPS tethering complexes-coordinators of endosome and lysosome fusion," Journal of Cell Science, vol. 126, no. 6, pp. 1307-1316, 2013.

[80] G. Cantalupo, P. Alifano, V. Roberti, C. B. Bruni, and C. Bucci, "Rab-interacting lysosomal protein (RILP): the Rab7 effector required for transport to lysosomes," The EMBO Journal, vol. 20, no. 4, pp. 683-693, 2001.

[81] I. Jordens, M. Fernandez-Borja, M. Marsman et al., "The Rab7 effector protein RILP controls lysosomal transport by inducing the recruitment of dynein-dynactin motors," Current Biology, vol. 11, no. 21, pp. 1680-1685, 2001.

[82] S. Pankiv, E. A. Alemu, A. Brech et al., "FYCO1 is a Rab7 effector that binds to LC3 and PI3P to mediate microtubule plus end-directed vesicle transport," The Journal of Cell Biology, vol. 188, no. 2, pp. 253-269, 2010.

[83] K. Yamano, A. I. Fogel, C. Wang, A. M. van der Bliek, and R. J. Youle, "Mitochondrial Rab GAPs govern autophagosome biogenesis during mitophagy," Elife, vol. 3, article e01612, 2014.

[84] A. Lizaso, K. T. Tan, and Y. H. Lee, "Beta-adrenergic receptor-stimulated lipolysis requires the RAB7-mediated autolysosomal lipid degradation," Autophagy, vol. 9, no. 8, pp. 1228-1243, 2013.

[85] B. Schroeder, R. J. Schulze, S. G. Weller, A. C. Sletten, C. A. Casey, and M. A. McNiven, "The small GTPase Rab7 as a central regulator of hepatocellular lipophagy," Hepatology, vol. 61, no. 6, pp. 1896-1907, 2015.

[86] D. Carmona-Gutierrez, A. Zimmermann, and F. Madeo, "A molecular mechanism for lipophagy regulation in the liver," Hepatology, vol. 61, no. 6, pp. 1781-1783, 2015.

[87] R. J. Schulze, K. Rasineni, S. G. Weller et al., "Ethanol exposure inhibits hepatocyte lipophagy by inactivating the small guanosine triphosphatase Rab7," Hepatology Communications, vol. 1, no. 2, pp. 140-152, 2017.

[88] S. Schuck, M. J. Gerl, A. Ang et al., "Rab10 is involved in basolateral transport in polarized Madin-Darby canine kidney cells," Traffic, vol. 8, no. 1, pp. 47-60, 2007.

[89] H. Sano, W. G. Roach, G. R. Peck, M. Fukuda, and G. E. Lienhard, "Rab10 in insulin-stimulated GLUT4 translocation," Biochemical Journal, vol. 411, no. 1, pp. 89-95, 2008.

[90] A. R. English and G. K. Voeltz, "Rab10 GTPase regulates ER dynamics and morphology," Nature Cell Biology, vol. 15, no. 2, p. 169, 2013.

[91] Z. Li, R. J. Schulze, S. G. Weller et al., "A novel Rab10EHBP1-EHD2 complex essential for the autophagic engulfment of lipid droplets," Science Advances, vol. 2, no. 12, article e1601470, 2016.

[92] C. Wang, Z. Liu, and X. Huang, "Rab32 is important for autophagy and lipid storage in Drosophila," PLoS One, vol. 7, no. 2, article e32086, 2012.

[93] Q. Li, J. Wang, Y. Wan, and D. Chen, "Depletion of Rab32 decreases intracellular lipid accumulation and induces lipolysis through enhancing ATGL expression in hepatocytes," Biochemical and Biophysical Research Communications, vol. 471, no. 4, pp. 492-496, 2016.

[94] M. R. Pulido, A. Diaz-Ruiz, Y. Jiménez-Gómez et al., "Rab18 dynamics in adipocytes in relation to lipogenesis, lipolysis and obesity," PLoS One, vol. 6, no. 7, article e22931, 2011.

[95] S. Martin, K. Driessen, S. J. Nixon, M. Zerial, and R. G. Parton, "Regulated localization of Rab18 to lipid droplets: effects of lipolytic stimulation and inhibition of lipid droplet catabolism," Journal of Biological Chemistry, vol. 280, no. 51, pp. 42325-42335, 2005.

[96] S. Ozeki, J. Cheng, K. Tauchi-Sato, N. Hatano, H. Taniguchi, and T. Fujimoto, "Rab18 localizes to lipid droplets and induces their close apposition to the endoplasmic reticulum-derived membrane," Journal of Cell Science, vol. 118, no. 12, pp. 2601-2611, 2005.

[97] C. Li, X. Luo, S. Zhao et al., "COPI-TRAPPIIactivates Rab18 and regulates its lipid droplet association," The EMBO journal, vol. 36, no. 4, pp. 441-457, 2017.

[98] J. J. Kim, Z. Lipatova, and N. Segev, “TRAPP complexes in secretion and autophagy," Frontiers in Cell and Developmental Biology, vol. 4, p. 20, 2016.

[99] Z. Zhang, S. Zhao, Z. Yao et al., "Autophagy regulates turnover of lipid droplets via ROS-dependent Rab25 activation in hepatic stellate cell," Redox Biology, vol. 11, pp. 322-334, 2017.

[100] A. B. Birgisdottir, T. Lamark, and T. Johansen, "The LIR motif - crucial for selective autophagy," Journal of Cell Science, vol. 126, no. 15, pp. 3237-3247, 2013.

[101] M. Lazarou, D. A. Sliter, L. A. Kane et al., "The ubiquitin kinase PINK1 recruits autophagy receptors to induce mitophagy," Nature, vol. 524, no. 7565, pp. 309-314, 2015.

[102] T. N. Nguyen, B. S. Padman, and M. Lazarou, "Deciphering the molecular signals of PINK1/Parkin mitophagy," Trends in Cell Biology, vol. 26, no. 10, pp. 733-744, 2016.

[103] T. L. Thurston, G. Ryzhakov, S. Bloor, N. von Muhlinen, and F. Randow, "The TBK1 adaptor and autophagy receptor NDP52 restricts the proliferation of ubiquitin-coated bacteria," Nature Immunology, vol. 10, no. 11, pp. 1215-1221, 2009.

[104] T. L. Thurston, M. P. Wandel, N. von Muhlinen, A. g. Foeglein, and F. Randow, "Galectin 8 targets damaged vesicles for autophagy to defend cells against bacterial invasion," Nature, vol. 482, no. 7385, pp. 414-418, 2012.

[105] S. Pankiv, T. H. Clausen, T. Lamark et al., "p62/SQSTM1 Binds Directly to Atg8/LC3 to Facilitate Degradation of Ubiquitinated Protein Aggregates by Autophagy," Journal of Biological Chemistry., vol. 282, no. 33, pp. 24131-24145, 2007.

[106] Y. N. Rui, Z. Xu, B. Patel et al., "Huntingtin functions as a scaffold for selective macroautophagy," Nature Cell Biology, vol. 17, no. 3, pp. 262-275, 2015.

[107] M. Martinez-Vicente and A. M. Cuervo, "Autophagy and neurodegeneration: when the cleaning crew goes on strike," The Lancet Neurology, vol. 6, no. 4, pp. 352-361, 2007.

[108] D. Lohmann, J. Spandl, A. Stevanovic, M. Schoene, J. Philippou-Massier, and C. Thiele, "Monoubiquitination of ancient ubiquitous protein 1 promotes lipid droplet clustering," PLoS One, vol. 8, no. 9, article e72453, 2013.

[109] J. Spandl, D. Lohmann, L. Kuerschner, C. Moessinger, and C. Thiele, "Ancient Ubiquitous Protein 1 (AUP1) Localizes to Lipid Droplets and Binds the E2 Ubiquitin Conjugase G2 (Ube2g2) via Its G2 Binding Region," Journal of Biological Chemistry, vol. 286, no. 7, pp. 5599-5606, 2011.

[110] C. Ward, N. Martinez-Lopez, E. G. Otten et al., "Autophagy, lipophagy and lysosomal lipid storage disorders," Biochimica 
et Biophysica Acta (BBA)-Molecular and Cell Biology of Lipids, vol. 1861, no. 4, pp. 269-284, 2016.

[111] C. Settembre, R. de Cegli, G. Mansueto et al., "TFEB controls cellular lipid metabolism through a starvation-induced autoregulatory loop," Nature Cell Biology, vol. 15, no. 6, pp. 647658, 2013.

[112] J.Xiong, K. Wang, J. He, G. Zhang, D. Zhang, and F. Chen, “TFE3 alleviates hepatic steatosis through autophagy-induced lipophagy and PGC1 $\alpha$-Mediated fatty acid $\beta$-Oxidation," International Journal of Molecular Sciences, vol. 17, no. 3, p. 387, 2016.

[113] Y. Fujimoto, Y. Nakagawa, A. Satoh et al., "TFE3 controls lipid metabolism in adipose tissue of male mice by suppressing lipolysis and thermogenesis," Endocrinology, vol. 154, no. 10, pp. 3577-3588, 2013.

[114] R. Singh, Y. Xiang, Y. Wang et al., "Autophagy regulates adipose mass and differentiation in mice," The Journal of clinical investigation, vol. 119, no. 11, pp. 3329-3339, 2009.

[115] B. q. Song, Y. Chi, X. Li et al., "Inhibition of Notch signaling promotes the adipogenic differentiation of mesenchymal stem cells through autophagy activation and PTENPI3K/AKT/mTOR pathway," Cellular Physiology and Biochemistry, vol. 36, no. 5, pp. 1991-2002, 2015.

[116] C. Zhang, Y. He, M. Okutsu et al., "Autophagy is involved in adipogenic differentiation by repressesing proteasomedependent PPAR $\gamma 2$ degradation," American Journal of Physiology-Endocrinology and Metabolism, vol. 305, no. 4, pp. E530-E539, 2013.

[117] X. Xiong, R. Tao, R. A. DePinho, and X. C. Dong, "The autophagy-related gene 14 (Atg14) is regulated by forkhead box $\mathrm{O}$ transcription factors and circadian rhythms and plays a critical role in hepatic autophagy and lipid metabolism," Journal of Biological Chemistry, vol. 287, no. 46, pp. 3910739114, 2012.

[118] S. Seok, T. Fu, S. E. Choi et al., "Transcriptional regulation of autophagy by an FXR-CREB axis," Nature, vol. 516, no. 7529, pp. 108-111, 2014.

[119] Y. K. Seo, T. I. Jeon, H. K. Chong, J. Biesinger, X. Xie, and T. F. Osborne, "Genome-wide localization of SREBP-2 in hepatic chromatin predicts a role in autophagy," Cell metabolism, vol. 13, no. 4, pp. 367-375, 2011.

[120] H. Zhang, S. Yan, B. Khambu et al., "Dynamic MTORC1TFEB feedback signaling regulates hepatic autophagy, steatosis and liver injury in long-term nutrient oversupply," Autophagy, vol. 14, no. 10, pp. 1779-1795, 2018.

[121] Y. Li, P. Yang, L. Zhao et al., "CD36 plays a negative role in the regulation of lipophagy in hepatocytes through an AMPK-dependent pathway[S]," Journal of Lipid Research, vol. 60, no. 4, pp. 844-855, 2019.

[122] J. M. Lee, M. Wagner, R. Xiao et al., "Nutrient-sensing nuclear receptors coordinate autophagy," Nature, vol. 516, no. 7529, p. 112, 2014.

[123] C. W. Lin, H. Zhang, M. Li et al., "Pharmacological promotion of autophagy alleviates steatosis and injury in alcoholic and non-alcoholic fatty liver conditions in mice," Journal of Hepatology, vol. 58, no. 5, pp. 993-999, 2013.

[124] R. A. Sinha, S. H. You, J. Zhou et al., "Thyroid hormone stimulates hepatic lipid catabolism via activation of autophagy," The Journal of clinical investigation, vol. 122, no. 7, pp. 2428-2438, 2012.

[125] J. A. Rodriguez-Navarro, S. Kaushik, H. Koga et al., "Inhibitory effect of dietary lipids on chaperone-mediated autoph- agy," Proceedings of the National Academy of Sciences, vol. 109, no. 12, pp. E705-E714, 2012.

[126] H. S. Kim, V. Montana, H. J. Jang, V. Parpura, and J. Kim, "Epigallocatechin Gallate (EGCG) Stimulates Autophagy in Vascular Endothelial Cells:A potential role for reducing lipid accumulation," Journal of Biological Chemistry, vol. 288, no. 31, pp. 22693-22705, 2013.

[127] J. Zhou, B. L. Farah, R. A. Sinha et al., "Epigallocatechin-3gallate (EGCG), a green tea polyphenol, stimulates hepatic autophagy and lipid clearance," PLoS One, vol. 9, no. 1, article e87161, 2014.

[128] R. A. Sinha, B. L. Farah, B. K. Singh et al., "Caffeine stimulates hepatic lipid metabolism by the autophagy-lysosomal pathway in mice," Hepatology, vol. 59, no. 4, pp. 1366-1380, 2014.

[129] M. Parafati, A. Lascala, V. M. Morittu et al., "Bergamot polyphenol fraction prevents nonalcoholic fatty liver disease via stimulation of lipophagy in cafeteria diet-induced rat model of metabolic syndrome," The Journal of nutritional biochemistry, vol. 26, no. 9, pp. 938-948, 2015.

[130] Y. Zhang, M. 1. Chen, Y. Zhou et al., "Resveratrol improves hepatic steatosis by inducing autophagy through the cAMP signaling pathway," Molecular Nutrition \& Food Research, vol. 59, no. 8, pp. 1443-1457, 2015.

[131] N. K. Zenkov, A. V. Chechushkov, P. M. Kozhin, N. V. Kandalintseva, G. G. Martinovich, and E. B. Menshchikova, "Plant phenols and autophagy," Biochemistry (Moscow), vol. 81, no. 4, pp. 297-314, 2016.

[132] A. Dolganiuc, P. G. Thomes, W.-X. Ding, J. J. Lemasters, and T. M. Donohue, "Autophagy in Alcohol-Induced liver diseases," Alcoholism: Clinical and Experimental Research, vol. 36, no. 8, pp. 1301-1308, 2012.

[133] M. J. Czaja, "Function of autophagy in nonalcoholic fatty liver disease," Digestive Diseases and Sciences, vol. 61, no. 5, pp. 1304-1313, 2016.

[134] R. Chen, Q. Wang, S. Song, F. Liu, B. He, and X. Gao, "Protective role of autophagy in methionine-choline deficient dietinduced advanced nonalcoholic steatohepatitis in mice," European Journal of Pharmacology, vol. 770, pp. 126-133, 2016.

[135] G. Ji, Y. Wang, Y. Deng, X. Li, and Z. Jiang, "Resveratrol ameliorates hepatic steatosis and inflammation in methionine/choline-deficient diet-induced steatohepatitis through regulating autophagy," Lipids in health and disease, vol. 14, no. 1, p. 134, 2015.

[136] A. Alexaki, S. D. Gupta, S. Majumder et al., "Autophagy regulates sphingolipid levels in the liver [S]," Journal of Lipid Research, vol. 55, no. 12, pp. 2521-2531, 2014.

[137] J. A. Chavez and S. A. Summers, “A ceramide-centric view of insulin resistance," Cell Metabolism, vol. 15, no. 5, pp. 585594, 2012.

[138] W. L. Holland, J. T. Brozinick, L. P. Wang et al., "Inhibition of ceramide synthesis ameliorates glucocorticoid-, saturatedfat-, and obesity-induced insulin resistance," Cell metabolism, vol. 5, no. 3, pp. 167-179, 2007.

[139] M. B. Khawar, H. Gao, and W. Li, "Autophagy and lipid metabolism," in Autophagy: Biology and Diseases. Advances in Experimental Medicine and Biology, vol 1206, Z. H. Qin, Ed., pp. 359-374, Springer, Singapore, 2019.

[140] K. H. Kim, Y. T. Jeong, H. Oh et al., "Autophagy deficiency leads to protection from obesity and insulin resistance by inducing Fgf21 as a mitokine," Nature Medicine, vol. 19, no. 1, pp. 83-92, 2013. 
[141] D. Ma, M. M. Molusky, J. Song et al., “Autophagy deficiency by hepatic FIP200 deletion uncouples steatosis from liver injury in NAFLD," Molecular Endocrinology, vol. 27, no. 10, pp. 1643-1654, 2013.

[142] A. S. Rambold, S. Cohen, and J. Lippincott-Schwartz, "Fatty acid trafficking in starved cells: regulation by lipid droplet lipolysis, autophagy, and mitochondrial fusion dynamics," Developmental Cell, vol. 32, no. 6, pp. 678-692, 2015.

[143] W.-X. Ding, M. Li, X. Chen et al., "Autophagy reduces acute ethanol-induced hepatotoxicity and steatosis in mice," Gastroenterology, vol. 139, no. 5, pp. 1740-1752, 2010.

[144] Y. Lu and A. I. Cederbaum, "Autophagy protects against CYP2E1/chronic ethanol-induced hepatotoxicity," Biomolecules, vol. 5, no. 4, pp. 2659-2674, 2015.

[145] Y. Wang, R. Singh, Y. Xiang, and M. J. Czaja, "Macroautophagy and chaperone-mediated autophagy are required for hepatocyte resistance to oxidant stress," Hepatology, vol. 52, no. 1, pp. 266-277, 2010.

[146] B. N. Reid, G. P. Ables, O. A. Otlivanchik et al., "Hepatic Overexpression of Hormone-sensitive Lipase and Adipose Triglyceride Lipase Promotes Fatty Acid Oxidation, Stimulates Direct Release of Free Fatty Acids, and Ameliorates Steatosis," Journal of Biological Chemistry, vol. 283, no. 19, pp. 13087-13099, 2008.

[147] C. P. Day, "Non-alcoholic steatohepatitis (NASH): where are we now and where are we going?," Gut, vol. 50, no. 5, pp. 585-588, 2002.

[148] C. P. Day and O. F. James, "Steatohepatitis: a tale of two "hits"?," Gastroenterology, vol. 114, no. 4, pp. 842-845, 1998.

[149] M. Amir, E. Zhao, L. Fontana et al., "Inhibition of hepatocyte autophagy increases tumor necrosis factor-dependent liver injury by promoting caspase- 8 activation," Cell Death and Differentiation, vol. 20, no. 7, pp. 878-887, 2013.

[150] T. Zhang, J. Liu, S. Shen, Q. Tong, X. Ma, and L. Lin, “SIRT3 promotes lipophagy and chaperon-mediated autophagy to protect hepatocytes against lipotoxicity," Cell Death \& Differentiation, vol. 27, no. 1, pp. 329-344, 2020.

[151] S. Carotti, K. Aquilano, F. Zalfa et al., "Lipophagy impairment is associated with disease progression in NAFLD," Frontiers in Physiology, vol. 11, 2020.

[152] Y. Gao, W. Zhang, L.-Q. Zeng et al., "Exercise and dietary intervention ameliorate high-fat diet-induced NAFLD and liver aging by inducing lipophagy," Redox biology, vol. 36, p. $101635,2020$.

[153] D. Wiggins and G. F. Gibbons, "The lipolysis/esterification cycle of hepatic triacylglycerol. Its role in the secretion of very-low-density lipoprotein and its response to hormones and sulphonylureas," Biochemical Journal, vol. 284, no. 2, pp. 457-462, 1992.

[154] Z. Papackova, H. Dankova, E. Palenickova, L. Kazdova, and M. Cahova, "Effect of short-and long-term high-fat feeding on autophagy flux and lysosomal activity in rat liver," Physiological Research, vol. 61, p. S67, 2012.

[155] V. Škop, M. Cahová, Z. Papáčková et al., “Autophagy-lysosomal pathway is involved in lipid degradation in rat liver," Physiological Research, vol. 61, no. 3, pp. 287-297, 2012.

[156] D. J. Klionsky, F. C. Abdalla, H. Abeliovich et al., "Guidelines for the use and interpretation of assays for monitoring autophagy," Autophagy, vol. 8, no. 4, pp. 445-544, 2012.

[157] E. Yamada and R. Singh, "Mapping autophagy on to your metabolic radar,” Diabetes, vol. 61, no. 2, pp. 272-280, 2012.
[158] Y. Fukuo, S. Yamashina, H. Sonoue et al., "Abnormality of autophagic function and cathepsin expression in the liver from patients with non-alcoholic fatty liver disease," Hepatology Research, vol. 44, no. 9, pp. 1026-1036, 2014.

[159] J. Kashima, K. Shintani-Ishida, M. Nakajima et al., "Immunohistochemical study of the autophagy marker microtubuleassociated protein 1 light chain 3 in normal and steatotic human livers," Hepatology Research, vol. 44, no. 7, pp. 779787, 2014.

[160] M. V. Stankov, D. Panayotova-Dimitrova, M. Leverkus et al., "Autophagy inhibition due to thymidine analogues as novel mechanism leading to hepatocyte dysfunction and lipid accumulation," Aids, vol. 26, no. 16, pp. 1995-2006, 2012.

[161] H. W. Park, H. Park, I. A. Semple et al., "Pharmacological correction of obesity-induced autophagy arrest using calcium channel blockers," Nature communications, vol. 5, no. 1, p. $4834,2014$.

[162] N. Martinez-Lopez and R. Singh, "Autophagy and lipid droplets in the liver," Annual review of nutrition, vol. 35, pp. 215237, 2015.

[163] V. Hernández-Gea, Z. Ghiassi-Nejad, R. Rozenfeld et al., "Autophagy releases lipid that promotes fibrogenesis by activated hepatic stellate cells in mice and in human tissues," Gastroenterology, vol. 142, no. 4, pp. 938-946, 2012.

[164] L. F. Thoen, E. L. M. Guimarães, L. Dollé et al., "A role for autophagy during hepatic stellate cell activation," Journal of Hepatology, vol. 55, no. 6, pp. 1353-1360, 2011.

[165] F. Cingolani and M. J. Czaja, "Regulation and functions of autophagic lipolysis," Trends in Endocrinology \& Metabolism, vol. 27, no. 10, pp. 696-705, 2016.

[166] J. Kovsan, M. Blüher, T. Tarnovscki et al., "Altered autophagy in human adipose tissues in obesity," The Journal of Clinical Endocrinology \& Metabolism, vol. 96, no. 2, pp. E268-E277, 2011.

[167] A. Öst, K. Svensson, I. Ruishalme et al., “Attenuated mTOR signaling and enhanced autophagy in adipocytes from obese patients with type 2 diabetes," Molecular medicine, vol. 16, no. 7-8, pp. 235-246, 2010.

[168] Q. Liu, Y.-M. Wang, and H.-F. Gu, "Lipophagy in atherosclerosis," Clinica Chimica Acta, vol. 511, pp. 208-214, 2020.

[169] S. Zhaorigetu, Z. Yang, I. Toma, T. A. McCaffrey, and C. A. $\mathrm{Hu}$, "Apolipoprotein L6, Induced in Atherosclerotic Lesions, Promotes Apoptosis and Blocks Beclin 1-dependent Autophagy in Atherosclerotic Cells," Journal of Biological Chemistry, vol. 286, no. 31, pp. 27389-27398, 2011.

[170] A. M. Cuervo, "Autophagy and aging: keeping that old broom working," Trends in Genetics, vol. 24, no. 12, pp. 604-612, 2008.

[171] A. Donati, A. Ventruti, G. Cavallini et al., "In vivo effect of an antilipolytic drug (3,5' -dimethylpyrazole) on autophagic proteolysis and autophagy-related gene expression in rat liver," Biochemical and Biophysical Research Communications, vol. 366, no. 3, pp. 786-792, 2008.

[172] T. Saitoh and S. Akira, "Regulation of innate immune responses by autophagy-related proteins," The Journal of Cell Biology, vol. 189, no. 6, pp. 925-935, 2010.

[173] S. Straniero, G. Cavallini, A. Donati et al., "Stimulation of autophagy by antilipolytic drugs may rescue rodents from age-associated hypercholesterolemia," Rejuvenation Research, vol. 12 , no. 2 , pp. $77-84,2009$. 
[174] R. Singh and A. M. Cuervo, "Lipophagy: connecting autophagy and lipid metabolism," International Journal of Cell Biology, vol. 2012, Article ID 282041, 12 pages, 2012.

[175] M. G. Metcalf, R. Higuchi-Sanabria, G. Garcia, C. K. Tsui, and A. Dillin, "Beyond the cell factory: homeostatic regulation of and by the UPRER," Science Advances, vol. 6, no. 29, article eabb9614, 2020.

[176] R. C. Taylor and A. Dillin, "XBP-1 is a cell-nonautonomous regulator of stress resistance and longevity," Cell, vol. 153, no. 7, pp. 1435-1447, 2013.

[177] J. R. Daniele, R. Higuchi-Sanabria, J. Durieux et al., "UPRERpromotes lipophagy independent of chaperones to extend life span," Science Advances, vol. 6, no. 1, 2020.

[178] J. R. Daniele, R. Higuchi-Sanabria, V. Ramachandran et al., "A non-canonical arm of UPRER mediates longevity through ER remodeling and lipophagy," BioRxiv, no. article 471177, 2018.

[179] M. Ait-Goughoulte, T. Kanda, K. Meyer, J. S. Ryerse, R. B. Ray, and R. Ray, "Hepatitis C virus genotype 1a growth and induction of autophagy," Journal of Virology, vol. 82, no. 5, pp. 2241-2249, 2008.

[180] M. Dreux, P. Gastaminza, S. F. Wieland, and F. V. Chisari, "The autophagy machinery is required to initiate hepatitis C virus replication," Proceedings of the National Academy of Sciences, vol. 106, no. 33, pp. 14046-14051, 2009.

[181] D. Sir, Y. Tian, W. 1. Chen, D. K. Ann, T. S. B. Yen, and J. H. J. Ou, "The early autophagic pathway is activated by hepatitis B virus and required for viral DNA replication," Proceedings of the National Academy of Sciences, vol. 107, no. 9, pp. 43834388, 2010.

[182] H. Tang, L. da, Y. Mao et al., "Hepatitis B virus X protein sensitizes cells to starvation-induced autophagy via upregulation of beclin 1 expression," Hepatology, vol. 49, no. 1, pp. 60-71, 2009.

[183] J. McLauchlan, "Lipid droplets and hepatitis C virus infection," Biochimica et Biophysica Acta (BBA)-Molecular and Cell Biology of Lipids, vol. 1791, no. 6, pp. 552-559, 2009.

[184] M. M. Samsa, J. A. Mondotte, N. G. Iglesias et al., "Dengue virus capsid protein usurps lipid droplets for viral particle formation," PLoS pathogens, vol. 5, no. 10, article e1000632, 2009.

[185] N. S. Heaton and G. Randall, "Dengue virus-induced autophagy regulates lipid metabolism," Cell Host \& Microbe, vol. 8, no. 5, pp. 422-432, 2010.

[186] J. E. McLean, A. Wudzinska, E. Datan, D. Quaglino, and Z. Zakeri, "Flavivirus NS4A-induced Autophagy Protects Cells against Death and Enhances Virus Replication," Journal of Biological Chemistry, vol. 286, no. 25, pp. 22147-22159, 2011.

[187] M. Panyasrivanit, M. P. Greenwood, D. Murphy, C. Isidoro, P. Auewarakul, and D. R. Smith, "Induced autophagy reduces virus output in dengue infected monocytic cells," Virology, vol. 418, no. 1, pp. 74-84, 2011.

[188] K. Liu and M. J. Czaja, "Regulation of lipid stores and metabolism by lipophagy," Cell death and differentiation, vol. 20, no. 1, p. 3, 2013.
[189] K. Wang, S. Li, T. Worku, X. Hao, L. Yang, and S. Zhang, "Rab11a is required for porcine reproductive and respiratory syndrome virus induced autophagy to promote viral replication," Biochemical and Biophysical Research Communications, vol. 492, no. 2, pp. 236-242, 2017.

[190] J. Wang, J. Y. Liu, K. Y. Shao et al., "Porcine reproductive and respiratory syndrome virus activates lipophagy to facilitate viral replication through downregulation of NDRG1 expression," J Virol, vol. 93, no. 17, 2019. 